\title{
Laser Interference Lithography for Fabrication of Planar Scale Gratings for Optical Metrology
}

\author{
Yuki Shimizu $^{1}$ (1)
}

Received: 8 September 2020 / Revised: 25 October 2020 / Accepted: 6 November 2020 / Published online: 16 January 2021

(c) The Author(s) 2021

\begin{abstract}
Laser interference lithography is an attractive method for the fabrication of a large-area two-dimensional planar scale grating, which can be employed as a scale for multi-axis optical encoders or a diffractive optical element in many types of optical sensors. Especially, optical configurations such as Lloyd's mirror interferometer based on the division of wavefront method can generate interference fringe fields for the patterning of grating pattern structures at a single exposure in a stable manner. For the fabrication of a two-dimensional scale grating to be used in a planar/surface encoder, an orthogonal two-axis Lloyd's mirror interferometer, which has been realized through innovation to Lloyd's mirror interferometer, has been developed. In addition, the concept of the patterning of the two-dimensional orthogonal pattern structure at a single exposure has been extended to the non-orthogonal two-axis Lloyd's mirror interferometer. Furthermore, the optical setup for the non-orthogonal two-axis Lloyd's mirror interferometer has been optimized for the fabrication of a large-area scale grating. In this review article, principles of generating interference fringe fields for the fabrication of a scale grating based on the interference lithography are reviewed, while focusing on the fabrication of a two-dimensional scale grating for planar/surface encoders. Verification of the pitch of the fabricated pattern structures, whose accuracy strongly affects the performance of planar/surface encoders, is also an important task to be addressed. In this paper, major methods for the evaluation of a grating pitch are also reviewed.
\end{abstract}

Keywords Planar scale grating · Interference lithography · Lloyd's mirror interferometer · Interference fringe

\section{Introduction}

A diffraction grating is one of the most important optical components employed in many academic and industrial fields. Its dispersive characteristics have been used for spectroscopic measurement [1], and nowadays it is widely employed in state-of-the-art scientific applications such as the pulse compression of an ultra-short pulse laser [2-4], as well as in industrial products such as optical drives or head-up displays [5, 6]. In the manufacturing industry, the diffraction grating is an important optical element employed as a scale of an optical linear encoder for precision positioning $[7,8]$. The accuracy of the grating pitch, which is the distance between the neighboring pattern structures of the diffraction grating, directly affects the accuracy of

Yuki Shimizu

yuki.shimizu@nano.mech.tohoku.ac.jp

1 Department of Finemechanics, Tohoku University, 6-6-01, Aramaki Aza-Aoba, Aoba-ku, Sendai, Miyagi 980-8579, Japan displacement measurement in a linear encoder. Therefore, a scale grating requires high-precision manufacturing process, as well as the verification/calibration process.

In recent years, multi-axis planar/surface encoders capable of measuring multi-axis displacement with a single measurement laser beam are attracting attention [9-17]. Figure 1 shows some examples of the surface/planar encoders. In planar/surface encoders, a two-dimensional diffraction grating is employed as the scale for displacement measurement. The resolution of in-plane displacement measurement is determined by the pitch of the grating in a planar/surface encoder. Therefore, accurate fabrication of micrometric/submicrometric two-dimensional pattern structures is required for the achievement of a high resolution in a planar/surface encoder. In addition, uniformity of the pattern amplitude, as well as the symmetry of the pattern profile, are necessary to achieve a high diffraction efficiency. Furthermore, it is necessary to establish a method to fabricate two-dimensional grating pattern structures over a wide area since the measurement range of a planar/surface encoder is determined by the size of a scale grating. For precision measuring instruments such 
(a)

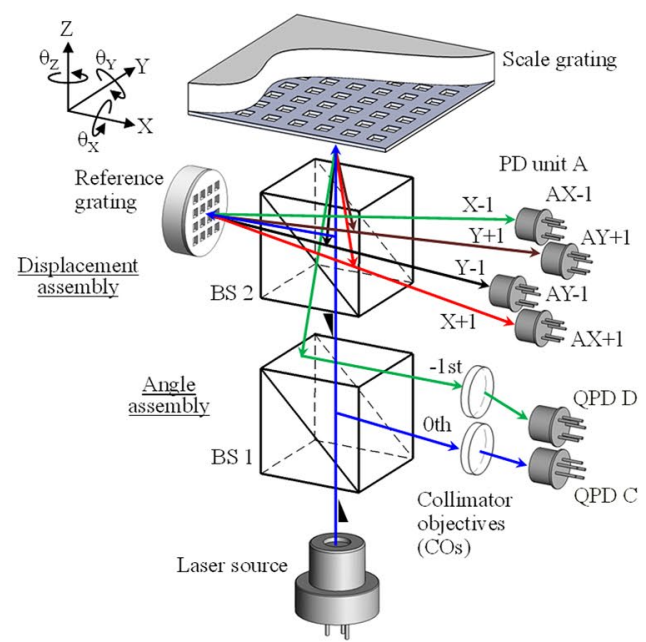

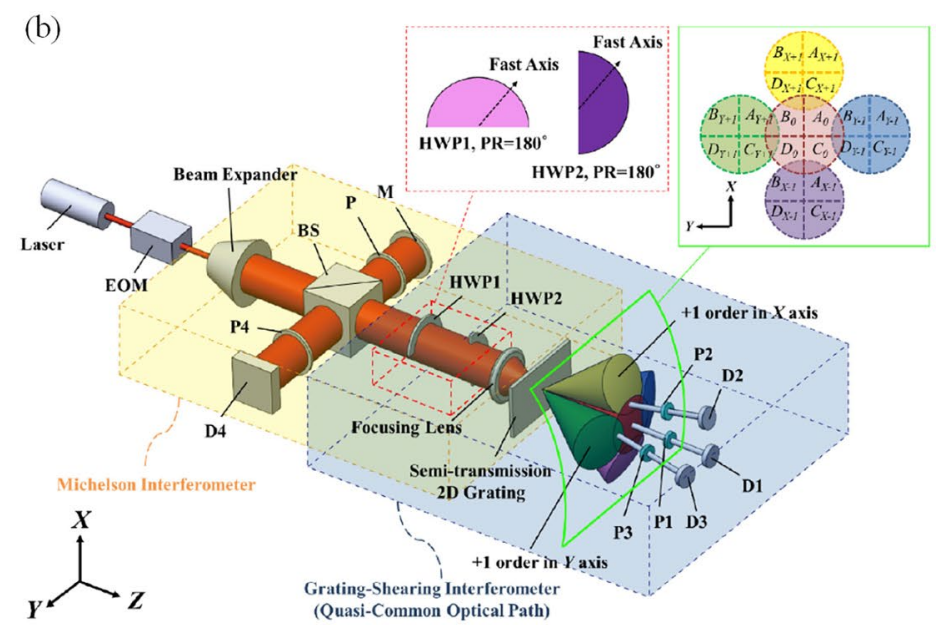

Fig. 1 Planar/surface encoders for multi-axis displacement measurement. a A six-degree-of-freedom surface encoder with a reflectivetype two-dimensional planar scale grating [9]. b A three-degree-of-

as micro coordinate measuring machines (micro CMMs) or scanning white-light interferometers, several-10 $\mathrm{mm}^{2}$ inplane measuring area is enough [18-21]. On the other hand, precision positioning systems to be employed in the semiconductor industry require a measuring area over $300 \mathrm{~mm}^{2}$ [22]; the fabrication of such a huge two-dimensional diffraction scale grating is not practical. To address the issue, a method employing a multi-beam optical head and a mosaic-scale grating composed of small two-dimensional diffraction scale gratings aligned in a matrix has been proposed [23-25]. This method can expand the in-plane measuring area of the planar/ surface encoder while reducing the influences of the gravitational deformation of the 2D scale grating and its manufacturing cost. Meanwhile, regarding the influence of cumulative error due to the stitching process, the size of each small 2D scale grating is preferably as large as possible.

Table 1 summarizes major fabrication methods for scale gratings used in the modern manufacturing industry. It is well known that the fabrication accuracy of scale gratings has been dramatically improved with the invention of the ruling engines and their improvements [26-29]. Even now many replica diffraction gratings are provided based on master gratings fabricated by the ruling engine. With the advancement of the fast-tool-servo (FTS) technology associated with the precision positioning, as well as the improvement of a diamond cutting tool, the fabrication of grating pattern structures having a complex profile has been achieved in the modern ultra-precision machining [30-32]. On the other hand, it takes time to carry out the machining of a diffraction grating over a large area and the long machining time induces the degradation of the machining accuracy due to the tool wear [33]. Ghost images and stray lights induced by the cutting mark on a machined surface are also issues to be addressed. Furthermore,

freedom planar encoder with a transparent-type two-dimensional planar scale grating [14]

the fabrication of narrow grating pattern structures with a micrometric or sub-micrometric grating pitch often employed in current applications is difficult by the mechanical machining. On the other hand, photolithography and laser interference lithography can fabricate narrow grating pattern structures, which cannot be fabricated by conventional mechanical machining, over a large area in a short manufacturing time [34, 35]. It should be noted that the fabrication of a grating by the photolithography process requires a precision photomask, as well as lithographic equipment; this leads to the increase of fabrication cost. On the contrary, laser interference lithography [36-40], which has been attracting attention since the invention of a laser source, can fabricate grating pattern structures without using a photomask. Although the fabrication of grating pattern structures with complex profiles is difficult, the features of mask-less and low-cost grating fabrication of the laser interference lithography are suitable for the fabrication of a large two-dimensional scale grating for multi-axis planar/ surface encoders and other optical sensors [41].

It should be noted that the pitch accuracy of a scale grating directly affects the measurement accuracy of an optical encoder system where the grating pattern structures on the scale are employed as the graduation for measurement. Therefore, it is necessary to establish a method to verify and calibrate the grating pitch of a fabricated scale grating. The state-of-the-art ultra-precision measuring instruments such as critical-dimension scanning electron microscopes (CD-SEMs) [42-46] or critical-dimension atomic force microscopes (CDAFM) [47-53] can directly evaluate the distance between the two neighboring grating pattern structures (namely, grating pitch) from the obtained pattern profile. Meanwhile, although some attempts have been made to expand the measurement range $[18,54-56]$ and improve measurement throughput $[57$, 
Table 1 Major methods for the fabrication of a diffraction grating

\begin{tabular}{ll}
\hline Mechanical fabrication & Pros \\
Complicated pattern structure can be fabricated with the enhancement of the fast-tool-servo (FTS) technology \\
Cons \\
Tool wear could degrade the quality of fabricated pattern structures \\
A minimum grating pitch is limited by the cutting tool to be employed (usually up to several micrometers) \\
Long machining time is required for the fabrication of pattern structures over a large area \\
Photolithography/direct writing & Pros \\
by electron beam (EB) & A high-resolution patterning possible with the enhancement of semiconductor technologies \\
& Pattern structures can be fabricated over a large area through repetitive lithography process \\
& Complicated structures can be fabricated with the controlled pattern exposure/development process \\
& Cons \\
Need to prepare for a precision mask pattern (EB does not require a mask pattern) & A high fabrication cost \\
Long fabrication time (in the case of direct writing by EB) \\
Pros \\
Pattern structures can be fabricated over a large area in a single exposure \\
A narrow grating pitch: up to a half of the laser wavelength \\
A low fabrication cost \\
Cons \\
Complicated pattern structures cannot be fabricated \\
\end{tabular}

58], it is still not a realistic way to evaluate the whole length/ area of a scale grating due to the low measurement throughput and limited measuring range. Many efforts have thus been made so far to realize fast evaluation of the grating pitch over a large measuring range based on some optical methods such as laser diffractometry [50, 59] or laser interferometry [60, 61].

In this paper, some techniques based on the laser interference lithography for the fabrication of two-dimensional orthogonal diffraction grating, which is employed as the scale in multi-axis planar/surface encoders, are reviewed. After brief descriptions of the principle of the laser interference lithography, two types of beam-dividing systems based on the division of amplitude method and the division of wavefront method are explained. After that, some multibeam interference lithography systems are explained, while focusing on the two types of multi-beam system based on the principle of Lloyd's mirror interferometer. Furthermore, a review is also carried out for some major methods of verifying/calibrating the grating pitch. A new approach for realizing rapid calibration of a two-dimensional diffraction scale grating based on the Fizeau interferometer is also explained.

\section{Principle of the Interference Lithography: Two-Beam Interference}

In the two-beam laser interference lithography, as shown in Fig. 2, two plane wavefronts are superposed to generate line interference fringe patterns on a substrate covered with a thin photoresist layer. Through the development process after the pattern exposure, line pattern structures can be obtained. Now we consider the case where the two coherent laser beams with an identical wavelength $\lambda$ are projected symmetrically onto a substrate with the angle of incidence of $\theta$. In the case, the intensity distribution $I$ of the obtained interference fringe patterns can be expressed as follows [62]:

$I=I_{1}+I_{2}+2 \sqrt{I_{1} I_{2}} \cos \phi$

where $I_{1}$ and $I_{2}$ are the intensities of two light beams, and $\phi$ is the phase difference between the two light beams at a particular $X$-position on the substrate that can be expressed by the following equation [62]:

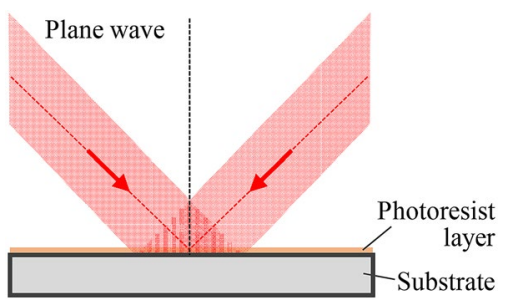

Fig. 2 Generation of line interference fringe patterns by the twobeam interference 
$\phi=\frac{2 \pi}{\lambda} x \cos 2 \theta$

The period of the interference line fringe patterns $g$, which corresponds to the pitch of grating pattern structures to be developed, can thus be obtained as follows:

$g=\frac{\lambda}{2 \sin \theta}$

As can be seen in Eq. (1), the light intensities of the two beams are required to be the same level so that high visibility of the interference fringe patterns can be obtained. High coherence is also required between the two beams. Spatial coherence of the two beams can be assured by employing a spatial filter composed of an objective lens and a pinhole, and generate the two beams from the laser beam passed through the spatial filter. In most of the cases of the laser interference lithography systems, sub-beams are generated from the same main laser beam. It should be noted that, regarding the temporal coherence, attention should be paid to choose the laser source. Denoting the wavelength width of the light source as $\Delta \lambda$, the coherence length $L_{\mathrm{c}}$ can be calculated as follows [62]:

$L_{\mathrm{c}}=\frac{\lambda^{2}}{\Delta \lambda}$

As can be seen in the above equation, a narrowerlinewidth laser with small $\Delta \lambda$ is required to achieve longer coherence length. It should be noted that high-contrast interference fringe patterns could be obtained with a low-cost laser having a wide linewidth when attention is paid for the design of interferometer while regarding the achievable coherence length $[63,64]$.

In the laser interference lithography, sub-beams are generated by dividing the main laser beam to assure the coherency between the two sub-beams. The phenomenon of interference can be classified into two types; the division of amplitude method [12, 36, 65-71] and the division of wavefront method [72-79]. Figure 3 shows some examples of the optical systems based on the division of amplitude method. In the optical setup shown in Fig. 3a, the main laser beam is split into two sub-beams by a beam splitter, and the two sub-beams are handled by several mirrors to generate interference fringes on the substrate [12]. One of the features of this optical configuration is the high degree of freedom in optical arrangement; a large-scale diffraction grating can easily be fabricated by expanding each of the sub-beams by a beam expander. However, this optical configuration is weak against external disturbances since non-common optical paths of the two sub-beams are long. This issue can be addressed by employing active control techniques for optical phase stabilization [80]; however, this makes the overall setup more complicated. Figure $3 \mathrm{~b}$ shows another example of the optical system based on the division of amplitude method. In the setup, a transparent-type grating with line pattern structures is employed to generate sub-beams (positive and negative first-order diffracted beams) from the main beam $[77,78]$. By superposing the two diffracted beams, line interference fringe patterns can be obtained. The transparent zeroth-order diffracted beam is stopped by using a hard stop. Although the degree-of-freedom of the optical arrangements could be slightly decreased, more stable interference lithography can be achieved by reducing the lengths of the non-common optical paths. (a)

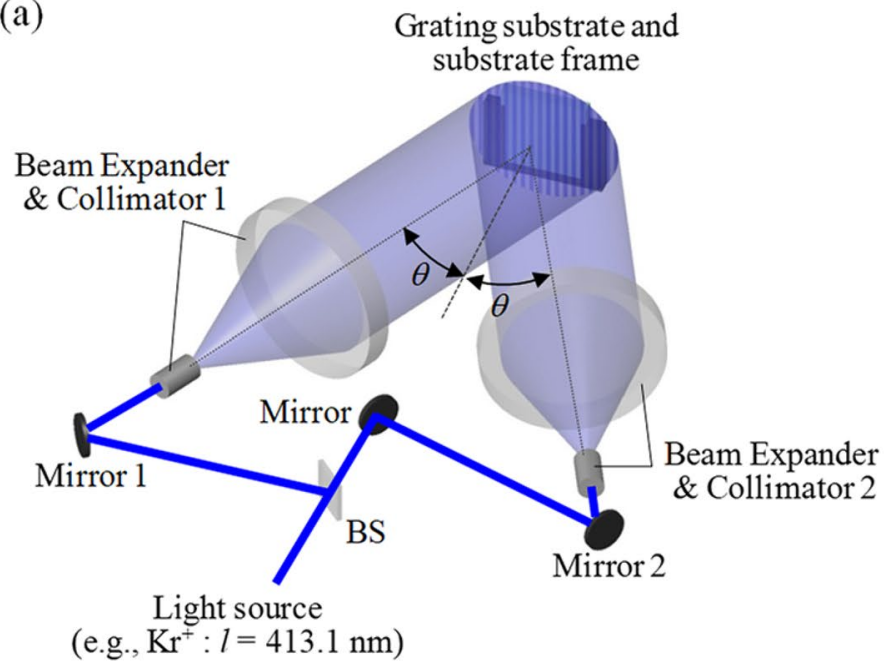

(b)

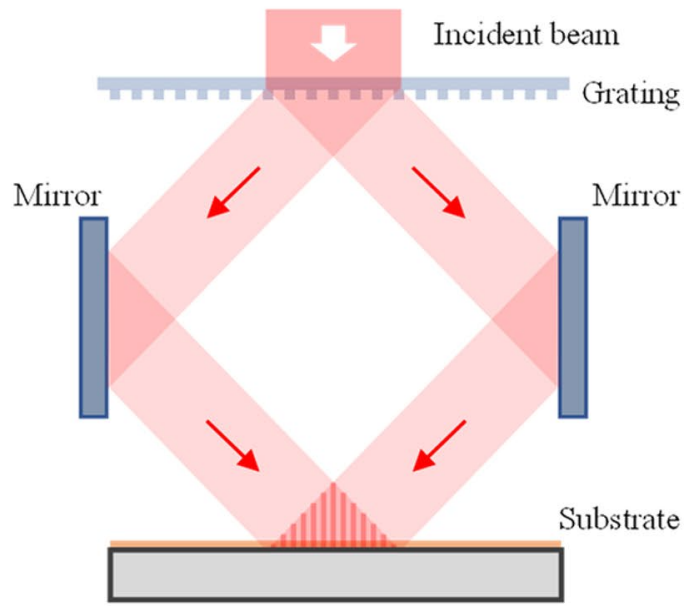

Fig. 3 Optical configurations for the laser interference lithography based on the division of amplitude method. a An optical configuration with a beam splitter for dividing the laser beam [12]. b An optical configuration with a transparent-type diffraction grating for dividing the laser beam 
Another method for generating the sub-beams is the division of wavefront method [72-79]. Figure 4a shows an example of the optical configurations based on the division of wavefront method [77]. In the optical setup, the positive and negative first-order diffracted beams generated at different areas on the same transparent-type grating are superposed on the substrate to generate line interference fringe patterns. Figure 4b shows another example of the setup [64] based on the division of wavefront method, which is referred to as Lloyd's mirror interferometer [72, 73]. A Lloyd's mirror is composed of a substrate and a mirror placed at a right angle to the substrate. A part of the main beam (direct-beam) is directly projected onto the substrate surface, while the other (reflected-beam) is projected onto the substrate surface after being reflected by the mirror. The superposed direct-beam and the reflected-beam generate line interference fringe patterns on the substrate surface. Although the degree-of-freedom of the optical arrangement is low, the

(a)

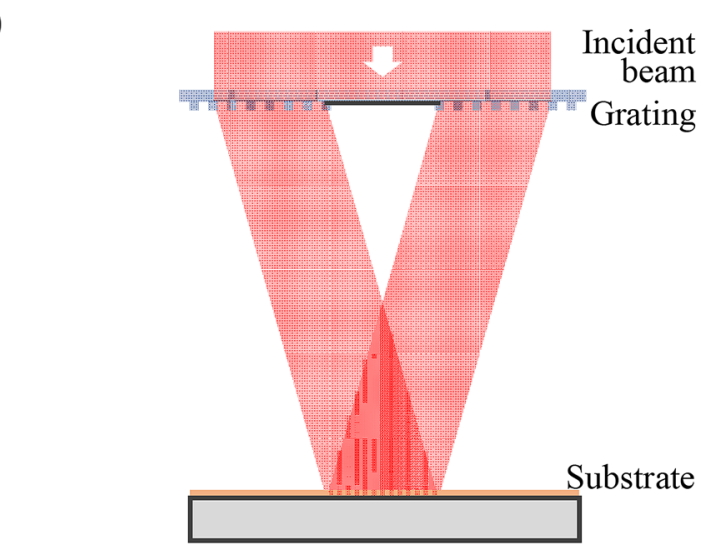

(b)

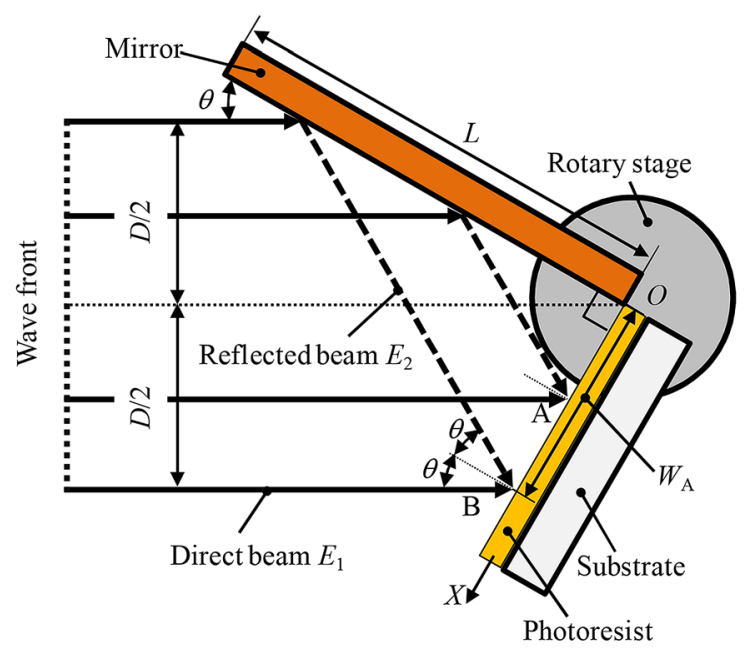

Fig. 4 Optical configurations for the laser interference lithography based on the division of wavefront method. a An optical configuration with a diffraction grating. b An optical configuration with a Lloyd's mirror interferometer [64] whole system can be constructed in a compact manner, while reducing the non-common optical paths between the directbeam and reflected-beam; these features of the Lloyd's mirror interferometer enables it to carry out more stable pattern exposure compared with the optical system based on the division of amplitude system. The period of line interference fringe patterns $g$ to be obtained by the Lloyd's mirror interferometer can be expressed by the following equation [64]:

$g=\frac{\lambda}{2 \sin \theta}$

where $\theta$ is the angle of incidence of the direct-beam projected onto the substrate in Lloyd's mirror interferometer. This equation is the same as Eq. (3) due to the geometric relationship of the two sub-beams in Lloyd's mirror interferometer.

The above-mentioned optical setups of the two-beam interference lithography can generate line interference fringe patterns. By carrying out pattern exposures twice before and after the $90^{\circ}$ rotation of a substrate, orthogonal two-dimensional grating pattern structures can be fabricated [74, 75]. However, the two-step exposure could induce the difference in the heights of $X$ - and $Y$-directional pattern structures as shown in Fig. 5, resulting in the difference of the $X$ - and $Y$-directional diffraction efficiencies; this could affect the performances of planar/surface encoders. This issue can be addressed by generating orthogonal two-dimensional interference fringe pattern through three- or four-beam interference, and carrying out pattern exposure by a single exposure process.

\section{Multi-beam Interference Lithography}

\subsection{Principle of the Multi-Beam Interference}

Interference between two sub-beams can generate line interference fringe patterns. As can be seen in Eq. (1), the interference fringe patterns have sinusoidal intensity distribution. In addition, the period of the interference fringe can be adjusted by changing the angle of incidence of the sub-beams. Regarding

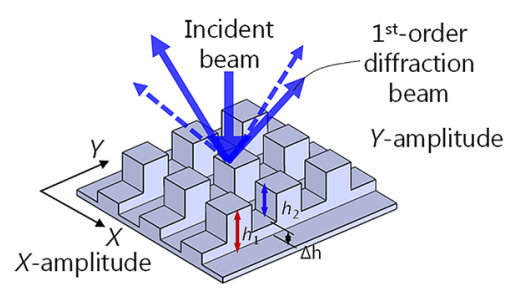

Fig. 5 A schematic of the two-dimensional grating pattern structures to be obtained through the development process after two-step line pattern exposure before and after $90^{\circ}$ rotation of the substrate 
the principle of Fourier transform, complex interference fringe patterns can be obtained by superposing several line interference fringe patterns. Furthermore, by superposing the several line interference fringe patterns extending in different directions, two-dimensional interference fringe patterns can be obtained. The complex amplitude of the sub-beam made incident to the substrate surface can be expressed by the following equation [62]:

$\overrightarrow{E_{n}}(\vec{r})=E_{n} \vec{e}_{n} \exp \left(\overrightarrow{k_{n}} \cdot \vec{r}+\varphi_{n}\right)$

where $\vec{r}$ is a position vector, $\overrightarrow{E_{n}}$ is the electric field amplitude, $\overrightarrow{e_{n}}$ is the unit vector of the polarization direction, and $\varphi_{n}$ is the initial phase. Interference fringe patterns by the three-beam interference can thus be expressed by the following equation [81]:

$I(\vec{r})=\sum_{i=1}^{3} E_{i}^{2}+2 \sum_{i=2}^{3} \sum_{k<i} E_{k} E_{i} \overrightarrow{e_{k}} \cdot \overrightarrow{e_{i}} \cos \left[\left(\overrightarrow{k_{k}}-\overrightarrow{k_{i}}\right) \cdot \vec{r}+\varphi_{k}-\varphi_{i}\right]$

By choosing the unit propagation vectors $\overrightarrow{k_{n}}$ (namely, adjusting the angles of incidence of the three sub-beams), interference fringe patterns and its periods can be controlled [82]. The period of the interference fringe patterns to be generated by the superposition of two sub-beams from the three can be expressed as follows [81]:

$g_{m, n}=\frac{2 \pi}{\left|\overrightarrow{k_{m}}-\overrightarrow{k_{n}}\right|}$

where $m<n \leq 3$ ( $m, n$ : natural numbers).

In the multi-beam interference lithography with the number of sub-beams larger than three, in most of the cases, all the sub-beams are generated from a single main laser beam. Figure 6 shows an example of the optical configurations for the multi-beam interference lithography based on the division of amplitude method [83]. A diffractive beam splitter (DBS) is employed to generate multiple diffracted beams, and some of them are chosen as the sub-beams by an aperture array to generate interference fringe patterns on the substrate. Since this configuration makes the alignments of sub-beams easy, many optical systems employing a diffractive optical element (DOE) for generating sub-beams have been developed so far [84-86].

Many multi-beam interference lithography systems based on the division of wavefront have also been reported [87-93], some examples of which are shown in Fig. 7. Figure 7a shows an example of the four-beam interference lithography system with a diffraction grating $[86,87,93]$. By generating four diffracted beams from the main laser beam with the enhancement of the four groups of grating patterns prepared on the same substrate, orthogonal two-dimensional interference fringe patterns can be obtained. However, the

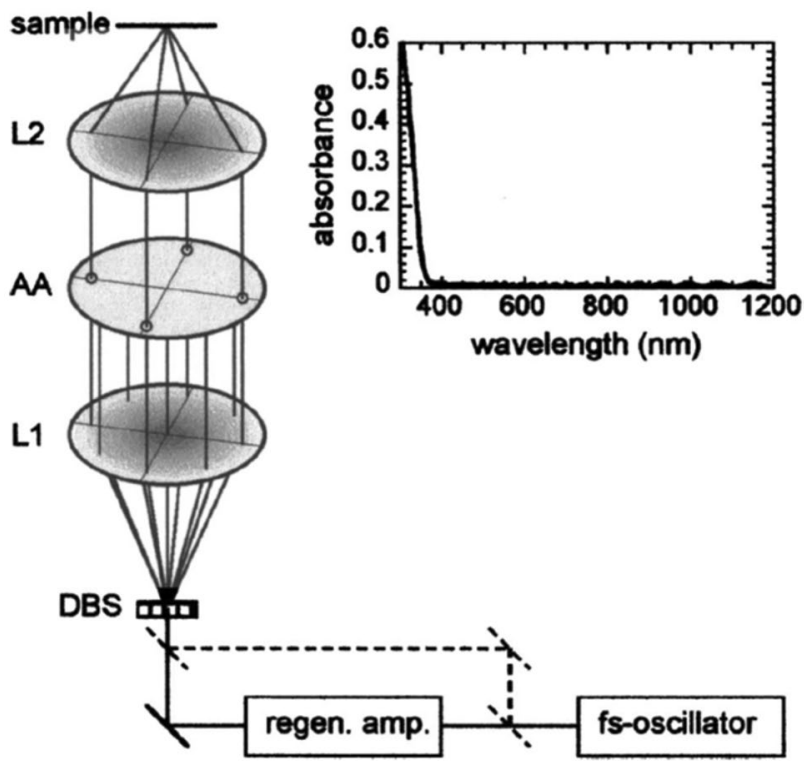

Fig. 6 An optical configuration for the multi-beam laser interference lithography based on the division of amplitude method with a diffractive beam splitter [83]

area of the interference fringe fields to be generated by this optical setup is limited by the size of the groups of grating patterns. Therefore, some additional techniques such as stitching lithography $[71,78]$ need to be employed for the fabrication of a large-area two-dimensional scale grating. Figure $7 \mathrm{~b}$ shows the three-beam interference lithography system composed of several optical fibers [89]. The main laser beam is made incident to a fiber bundle to generate sub-beams for multi-beam interference lithography. Due to the flexibility of the optical fibers, the angle of incidence of each sub-beam can easily be adjusted. In addition, it is easy to carry out the polarization modulation control of each of the sub-beams. However, it is not a realistic way to apply the optical configuration to the fabrication of a large-scale two-dimensional scale grating. On the other hand, a two-axis Lloyd's mirror interferometer, in which an additional mirror is applied to the conventional one-axis Lloyd's mirror interferometer, is a candidate to fabricate a two-dimensional scale grating over a large area $[81,88,90,91,94]$. Figure $7 \mathrm{c}$ shows a schematic of the two-axis Lloyd's mirror interferometer for the fabrication of the two-dimensional hexagonal pattern structures. In the setup, an angle between the two mirrors is adjusted to be $120^{\circ}$, while each of them is placed at a right angle with respect to the substrate [91]. On the substrate, the sub-beam directly made incident to the substrate and the sub-beams reflected by the corresponding mirrors are superposed with each other to generate hexagonal interference fringe patterns, as the consequence of the two line interference fringes having an angle of $120^{\circ}$ [91]. By adjusting an angle between the two mirrors to be $90^{\circ}$, 
(a)
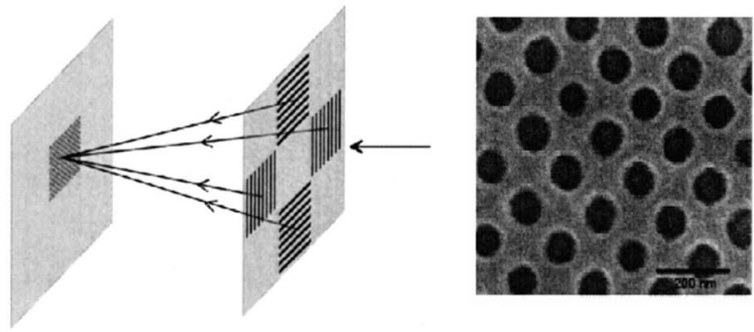

(b)
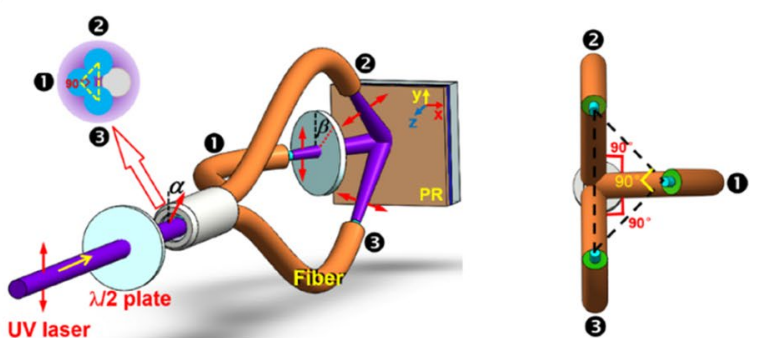

(c)

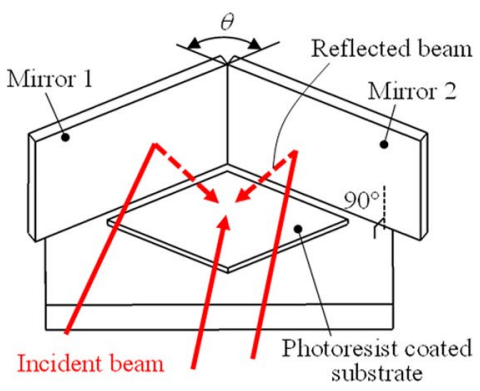

Fig. 7 An optical configuration for the multi-beam laser interference lithography based on the division of wavefront method. a Optical configuration with diffraction gratings [86]. b Optical configuration with a fiber bundle [89]. c Optical configuration based on the oneaxis Lloyd's mirror interferometer with an additional mirror

orthogonal two-dimensional interference fringe patterns can be generated for the fabrication of two-dimensional scale grating [90, 94-96], the details of which are explained in the following section.

\subsection{Orthogonal Two-Axis Lloyd's Mirror Interferometer}

\subsubsection{Basic Principle}

An orthogonal two-axis Lloyd's mirror interferometer can be constructed based on the one-axis Lloyd's mirror interferometer, a schematic of which is shown in Fig. 8a [95]. The main laser beam, whose propagation direction is parallel with the $X Z$-plane, is made incident to the interferometer composed of a substrate and the $X$-mirror. The direct sub-beam (Beam1) and the sub-beam reflected by the $X$-mirror (Beam2) are superposed on the substrate to generate line interference fringe patterns parallel with the $Y$-axis in Fig. 8a. In the orthogonal two-axis Lloyd's mirror interferometer shown in Fig. 8b, a major modification is made in such a way that an additional mirror ( $Y$-mirror) perpendicular to both the substrate and the $X$-mirror is added to the one-axis Lloyd's mirror interferometer to construct a corner-cube-like optical configuration, while the propagation direction of the main laser beam is set to be parallel with the meridian plane with the azimuthal angle $\varphi$ with respect to the $Y$-mirror as shown in Fig. 8c. In the optical configuration, the main laser beam can be divided into the following five sub-beams:

Beam1: Directly enters the substrate

Beam2: Enters the substrate after being reflected by the $X$-mirror

Beam3: Enters the substrate after being reflected by the $Y$-mirror

Beam4: Enters the substrate after being reflected by the $X$-mirror and then the $Y$-mirror

Beam4': Enters the substrate after being reflected by the $Y$-mirror and then the $X$-mirror

Interference between the Beam 1 and Beam2 generates line interference fringe patterns parallel with the $Y$-axis, while the interference between the Beam1 and Beam3 (a)

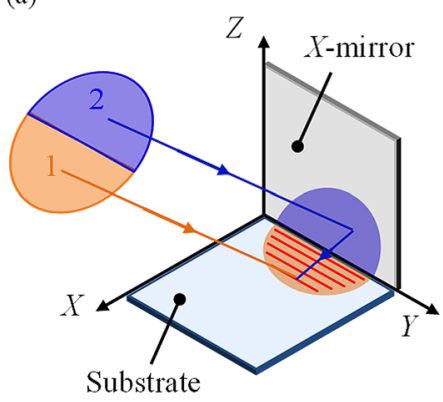

(b)

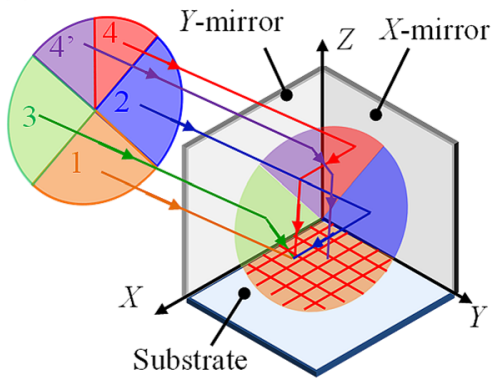

(c) Axis of the incident beam

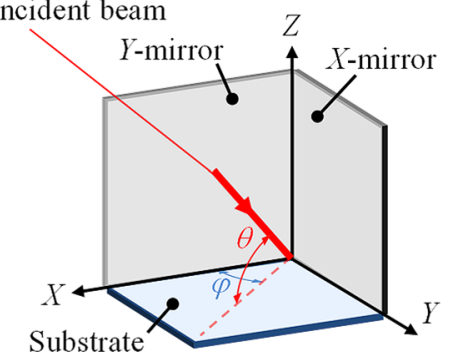

Fig. 8 Orthogonal two-axis Lloyd's mirror interferometer [95]. a Conventional one-axis Lloyd's mirror interferometer. b Orthogonal two-axis Lloyd's mirror interferometer with an additional mirror. $\mathbf{c}$ Definition of the azimuthal angle 
Table 2 Interference fringe patterns and their periods to be generated by each pair of beams [95]

\begin{tabular}{|l|c|c|c|c|}
\hline & & & \\
\hline & $g_{12}=\lambda /(\sqrt{2} \cos \theta)$ & $g_{13}=\lambda /(\sqrt{2} \cos \theta)$ & & \\
\hline
\end{tabular}

generates the one parallel with the $X$-axis. As the consequence of the superposition of the two interference fringe patterns, the orthogonal two-dimensional interference fringe patterns can be obtained. Denoting the angle between the propagation direction of the $i$ th sub-beam and the substrate surface as $\theta_{i}$, the unit propagation vectors $\vec{k}_{i}$ of the $i$ th subbeam can be expressed as follows:

$\vec{k}_{1}=\frac{2 \pi}{\lambda}\left(\begin{array}{l}-\cos \theta_{1} \cos \varphi \\ -\cos \theta_{1} \sin \varphi \\ -\sin \theta_{1}\end{array}\right), \quad \vec{k}_{2}=\frac{2 \pi}{\lambda}\left(\begin{array}{l}\cos \theta_{2} \cos \varphi \\ -\cos \theta_{2} \sin \varphi \\ -\sin \theta_{2}\end{array}\right)$,

$\vec{k}_{3}=\frac{2 \pi}{\lambda}\left(\begin{array}{l}-\cos \theta_{3} \cos \varphi \\ \cos \theta_{3} \sin \varphi \\ -\sin \theta_{3}\end{array}\right), \quad \vec{k}_{4}=\frac{2 \pi}{\lambda}\left(\begin{array}{l}\cos \theta_{4} \cos \varphi \\ \cos \theta_{4} \sin \varphi \\ -\sin \theta_{4}\end{array}\right)$

Also, the light intensity distribution of the interference fringe patterns to be obtained by the four-beam interference can be obtained as follows:

$I(\vec{r})=\sum_{\ell=1}^{4} E_{\ell}^{2}+2 \sum_{m=2}^{4} \sum_{n<m} E_{m} E_{n} \overrightarrow{e_{m}} \cdot \overrightarrow{e_{n}} \cos \left[\left(\overrightarrow{k_{n}}-\overrightarrow{k_{m}}\right) \cdot \vec{r}+\varphi_{n}-\varphi_{m}\right]$

In the orthogonal two-axis Lloyd's mirror interferometer, the two mirrors are arranged to have a right angle with respect to the substrate. Therefore, in the ideal case without the misalignments of the optical components, $\theta_{1}=\theta_{2}=\theta_{3}=\theta_{4}$. Assuming that an azimuthal angle of $\varphi$ is $45^{\circ}$, the combination of two sub-beams from the four gives ${ }_{4} C_{2}=6$ types of interference fringe patterns as summarized in Table 2. As can be seen in the table, the line interference fringe patterns parallel with the $Y$-axis can be obtained by the interference between the Beam1 and Beam2, as well as that between Beam 3 and Beam4. The periods of both the line interference fringe patterns $g_{12}$ and $g_{34}$ are $g_{12}=g_{34}=\lambda /$ $(\sqrt{2} \cos \theta)$.

\subsubsection{Influences of Misalignments of Optical Components}

In the actual case of the setup, there should be some misalignment for each optical component, and it could affect the accuracy of fabricated two-dimensional grating pattern structures. Figure 9 shows a schematic diagram of the visible stripes in the interference fringe patterns to be generated in the case where the $X$-mirror has an angular misalignment about the $Z$-axis. The angular misalignment of the $X$-mirror give a slight change in the period of the line interference fringe patterns parallel with the $Y$-axis to be obtained by the interference between the Beam 3 and Beam4' $\left(g_{34^{\prime}}\right)$; this could result in a small period difference between $g_{12}$ and $g_{34}$, and generates Moiré-like visible stripes with a low spatial frequency on the fabricated two-dimensional pattern structures.

The misalignments of the angle of incidence $\theta$ and the azimuthal angle $\varphi$ also affect the two-dimensional interference fringe patterns. The angle of incidence $\theta$ only affects the period of the interference fringe patterns. It should be noted that the azimuthal angle misalignment could generate visible stripes with a low spatial frequency in the interference fringe patterns. Figure 10 shows the results of numerical calculations simulating the influence of the azimuthal 
(a)

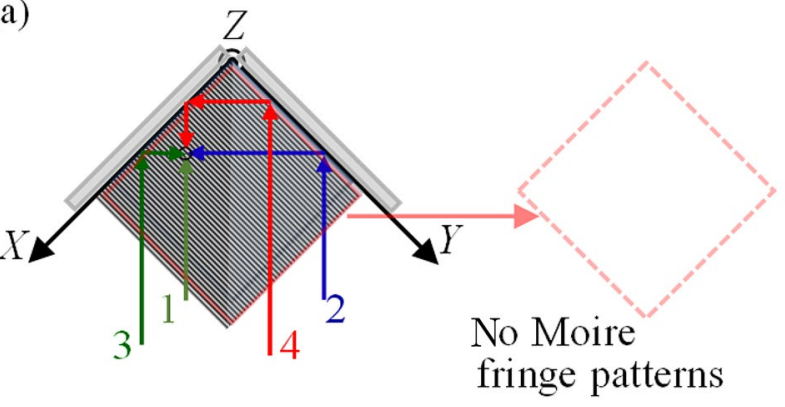

(b)

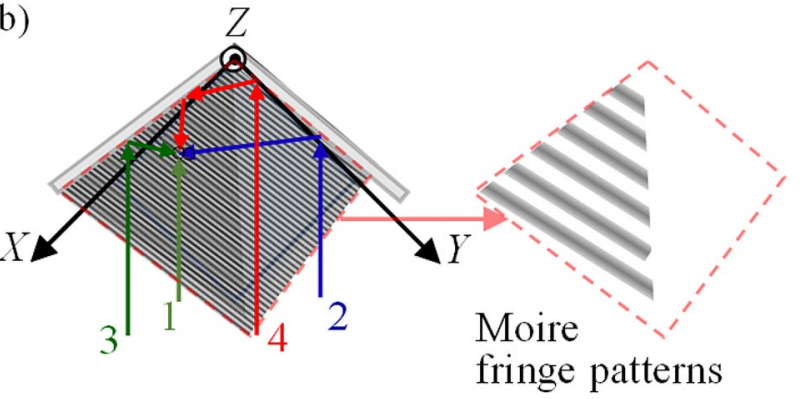

Fig. 9 Visible stripes in the interference fringe patterns due to the angular misalignment of the $Y$-mirror [95]. a Without the misalignment. b With the angular misalignment about the $Z$-axis

angle misalignment. As can be seen in these results, optical misalignments could strongly affect the interference fringe patterns to be generated by the orthogonal two-axis Lloyd's mirror interferometer. Meanwhile, regarding the principle of the generation of interference fringe patterns summarized in Table 2, the removal of Beam4 and Beam4' is effective in avoiding the occurrence of the visible stripes on the fabricated two-dimensional pattern structures. Figure 11 shows the optical setup for the orthogonal two-axis Lloyd's mirror interferometer, where a physical filter is placed in the interferometer unit to remove Beam4 and Beam4' [96]. Figure $12 \mathrm{a}, \mathrm{b}$ compares the two-dimensional grating pattern structures fabricated by the optical setup with and without the filtering of Beam4 and Beam4' [95]. In Fig. 12a, a photograph of the grating pattern structures exposed by the interference fringe patterns generated by the superposition of all the four beams (Beams1, 2, 3 and 4/4') is indicated. As can be seen in the figure, visible stripes due to the influence of optical misalignments can be observed; this can be overcome by filtering Beam4/4' by employing a physical filter as shown in Fig. 11c. Figure 12b shows the grating pattern structures exposed by the interference fringe patterns by the three beams (Beams1, 2 and 3). As can be seen in the figure, the occurrence of the visible stripes with a low spatial frequency has successfully been eliminated.

\subsubsection{Polarization Modulation Control}

The physical filtering of the Beam4 and Beam4' is effective in vanishing the visible stripes on the fabricated pattern structures. Meanwhile, the line interference fringe patterns as the consequence of the interference between the Beam2 and Beam3, which are extending in the direction of $45^{\circ}$ with respect to the $X$-axis as can be seen in Table 2, are not necessary for the fabrication of the orthogonal two-dimensional grating pattern structures. It is thus necessary to reduce the influence of these fringe patterns. A polarization modulation control is effective in reducing the unnecessary interference fringe patterns $[88,89,97]$. Figure 13 summarizes the interference fringe patterns estimated in numerical calculations for each of the cases with different polarization modulation

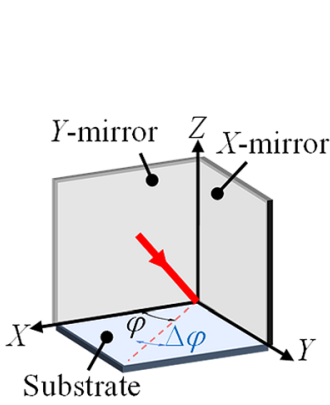

(a)
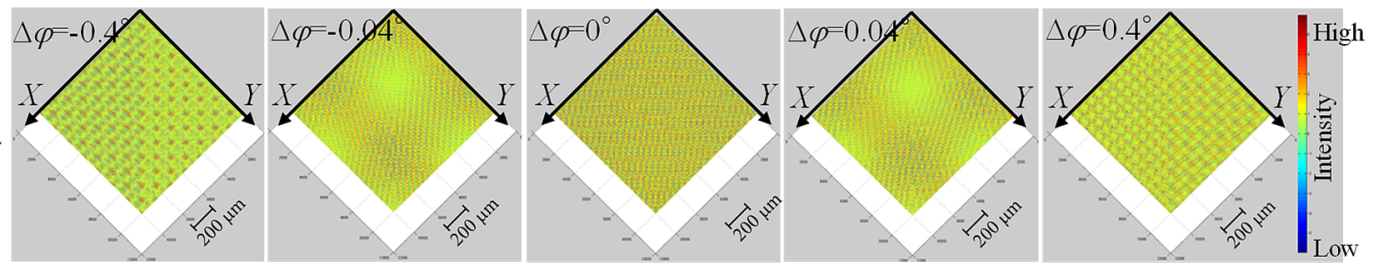

(b)

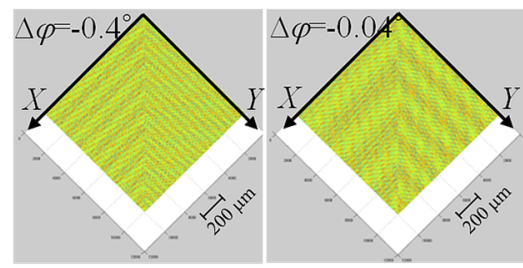

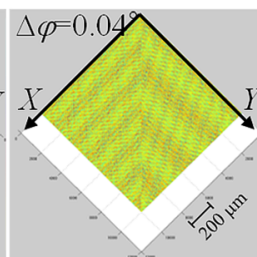

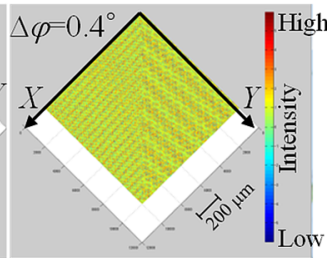

(c)
Fig. 10 Influences of the azimuthal angle misalignment of the main laser beam $\Delta \varphi$ in the orthogonal two-axis Lloyd's mirror interferometer [95]. a Azimuthal angle misalignment of the main laser beam. b Estimated interference fringe patterns without the other optical misalignments except $\Delta \varphi$. c Estimated interference fringe patterns with misalignments of $\Delta \varphi_{X Y}=0.08^{\circ}, \Delta \varphi_{Y X}=0.08^{\circ}, \Delta \varphi_{X Z}=0.4^{\circ}$, $\Delta \varphi_{Y Z}=0.4^{\circ}, \Delta \varphi=0^{\circ}$ 


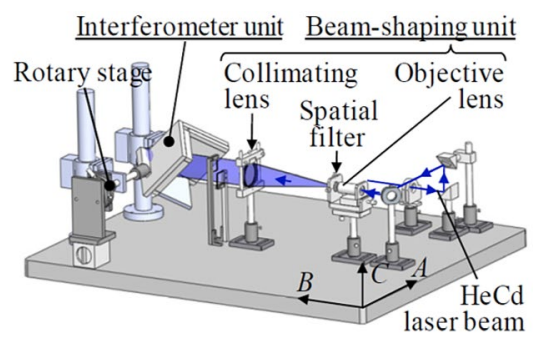

(a)

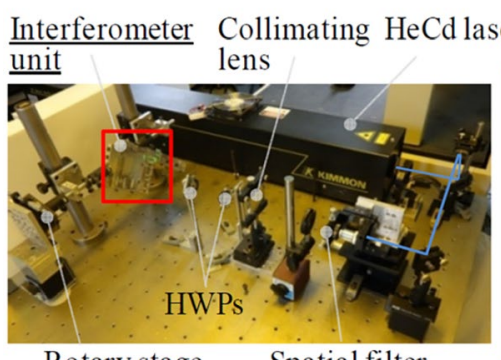

Rotary stage Spatialfilter

(b)

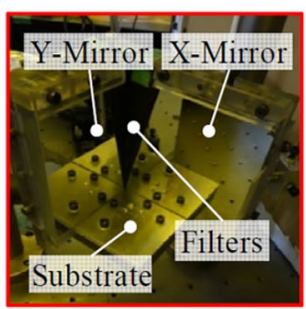

(c)
Fig. 11 Orthogonal two-axis Lloyd's mirror interferometer [96]. a A schematic of the setup. b A photograph of the setup. $\mathbf{c}$ The orthogonal two-axis interference unit (a)

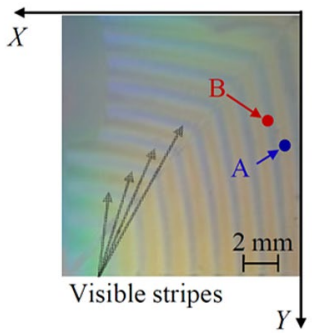

(b)

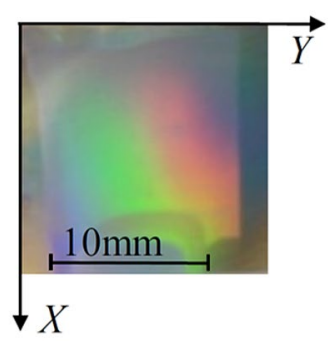

Fig. 12 Photographs of the fabricated two-dimensional scale gratings [95]. a A scale grating with the visible stripes due to the optical misalignments in the orthogonal two-axis Lloyd's mirror interferometer. b A scale grating fabricated by using the orthogonal two-axis interference unit shown in Fig. 11c

control states [96]. Microscopic images of the fabricated grating pattern structures measured by an AFM are also shown in the figure. As can be seen in the figure, polarization states of each sub-beam could strongly affect the interference fringe patterns, and it is necessary to optimize the polarization states of the sub-beams for obtaining twodimensional grating pattern structures without distortion. It should be noted that attention should be paid to the change in the polarization states of the sub-beams after the reflection by the $X$-and $Y$-mirrors in the interferometer unit. It is well known that most of the commercially-available dielectric mirrors are composed of multiple thin layers, and the material and thickness of each layer could affect the polarization state of the reflected sub-beam [62]. Therefore, it is necessary to take the influence of $X$ - and $Y$-mirrors into consideration for the optimization of the polarization modulation control in the orthogonal two-axis Lloyd's mirror interferometer [96]. Numerical calculations have revealed that it is difficult to vanish the influence of unnecessary interference fringe patterns; meanwhile, it has also been revealed that the orthogonal two-axis Lloyd's mirror interferometer in optimized polarization states could generate affordable orthogonal two-dimensional interference fringe patterns as shown in Fig. 14 [96].

For the fabrication of a large-area two-dimensional scale grating based on the orthogonal two-axis Lloyd's mirror interferometer, a large-scale interferometer unit is required. However, as can be seen in the estimated interference fringe patterns shown in Fig. 10, the angular position of the interferometer unit with respect to the main laser beam should be adjusted precisely. However, it is not an easy task to carry out the precise alignment of a large-scale interferometer unit.

\subsection{Non-Orthogonal Two-Axis Lloyd's Mirror Interferometer}

\subsubsection{Basic Principle}

For the fabrication of a two-dimensional scale grating with an optical setup in a small dimension, a non-orthogonal two-axis Lloyd's mirror interferometer has been developed. Figure $15 \mathrm{a}, \mathrm{b}$ are schematics of the interference lithography units of the orthogonal-type and the nonorthogonal-type [88, 98]. In the orthogonal-type interference lithography unit, a pair of orthogonal mirrors are placed at a right angle with respect to the substrate, while the main laser beam is projected onto the interferometer unit with an oblique angle of incidence. On the contrary, in the non-orthogonal-type interference lithography unit, the angles of $X$ - and $Y$-mirrors are set to be $90^{\circ}+\theta_{X}$ and $90^{\circ}+\theta_{Y}\left(\theta_{X}>0^{\circ}, \theta_{Y}>0^{\circ}\right)$, respectively, while the main laser beam is projected onto the substrate in the interferometer unit at a right angle. On the substrate surface, the two sub-beams reflected by each of the $X$ - and $Y$-mirrors and the direct-beam are superposed with each other to generate two-dimensional interference fringe patterns. This optical configuration could avoid the generation of visible stripes with a low spatial frequency on the fabricated pattern structures that can be observed in the orthogonal-type, since there are no multiple-reflected subbeams in the non-orthogonal type. In addition, the optical configuration of the non-orthogonal type with the main laser beam projected onto the substrate at a right angle makes the alignments of the whole setup easier than those for the orthogonal-type. Furthermore, the periods of the $X$ - and $Y$-directional interference fringe patterns can be adjusted 

patterns estimated in numerical calculations and fabricated grating pattern structures for each of the polarization status of the three-beams in the orthogonal two-axis Lloyd's mirror interferometer [96]
Fig. 13 Interference fringe
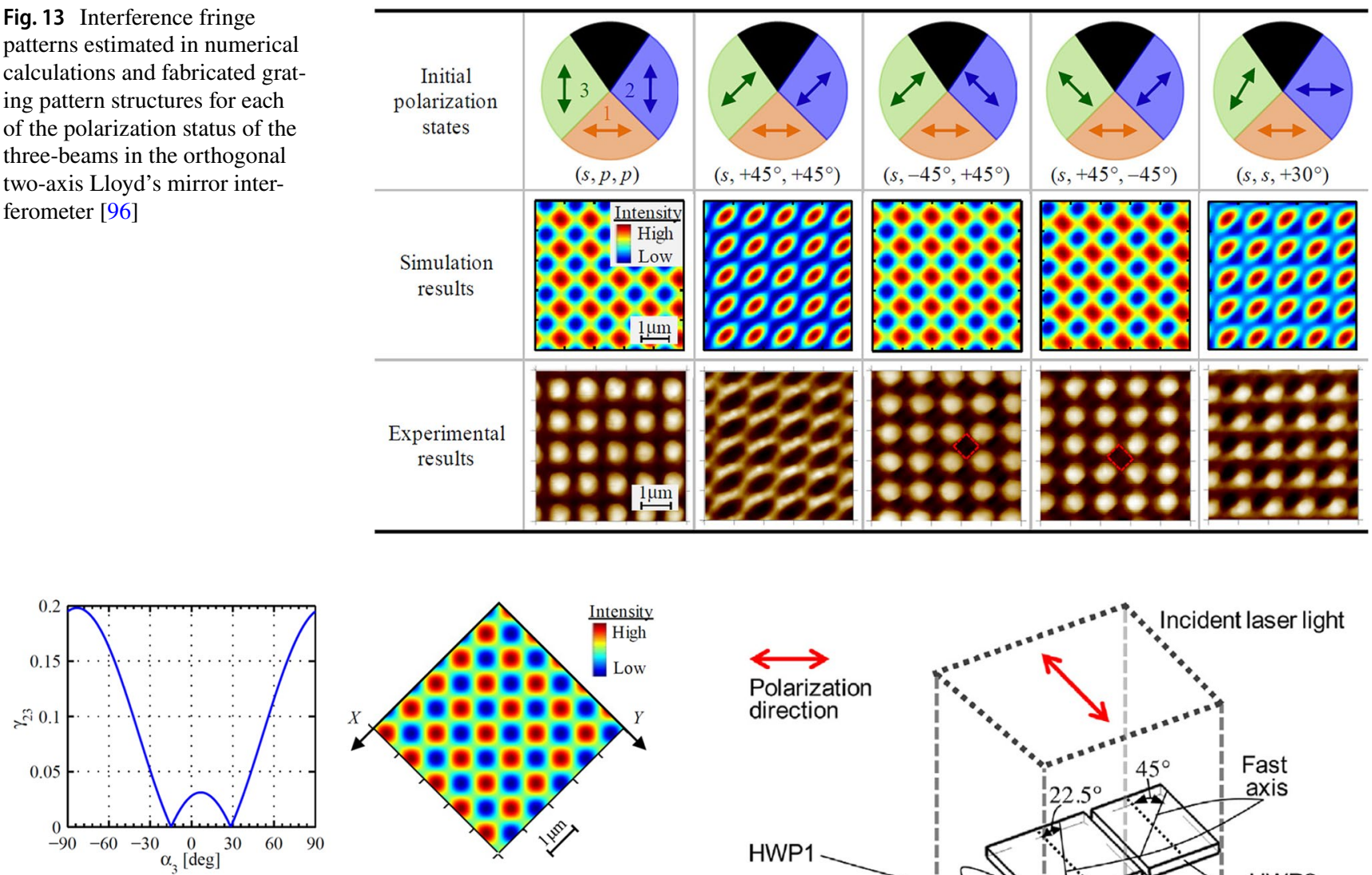

Fig. 14 A reduction of the unnecessary interference fringe components by the optimization of the polarization modulation control in the orthogonal two-axis Lloyd's mirror interferometer [96]

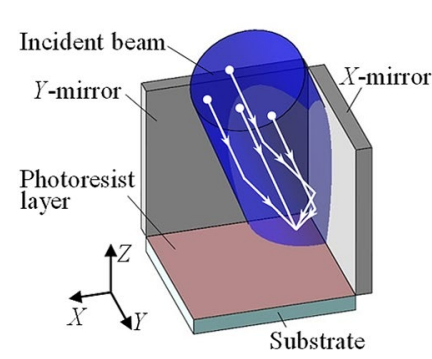

(a)

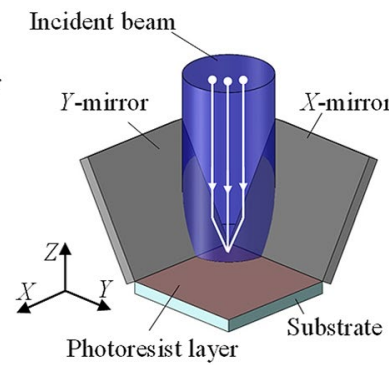

(b)
Fig. 15 Two-types of two-axis Lloyd's mirror interferometers for the fabrication of two-dimensional orthogonal grating pattern structures [98]. a Orthogonal type. b Non-orthogonal type

precisely and independently by adjusting the angles $\theta_{X}$ and $\theta_{Y}$ of the $X$ - and $Y$-mirrors, respectively.

Figure 16 shows the non-orthogonal two-axis Lloyd's mirror interferometer unit with a polarization modulation

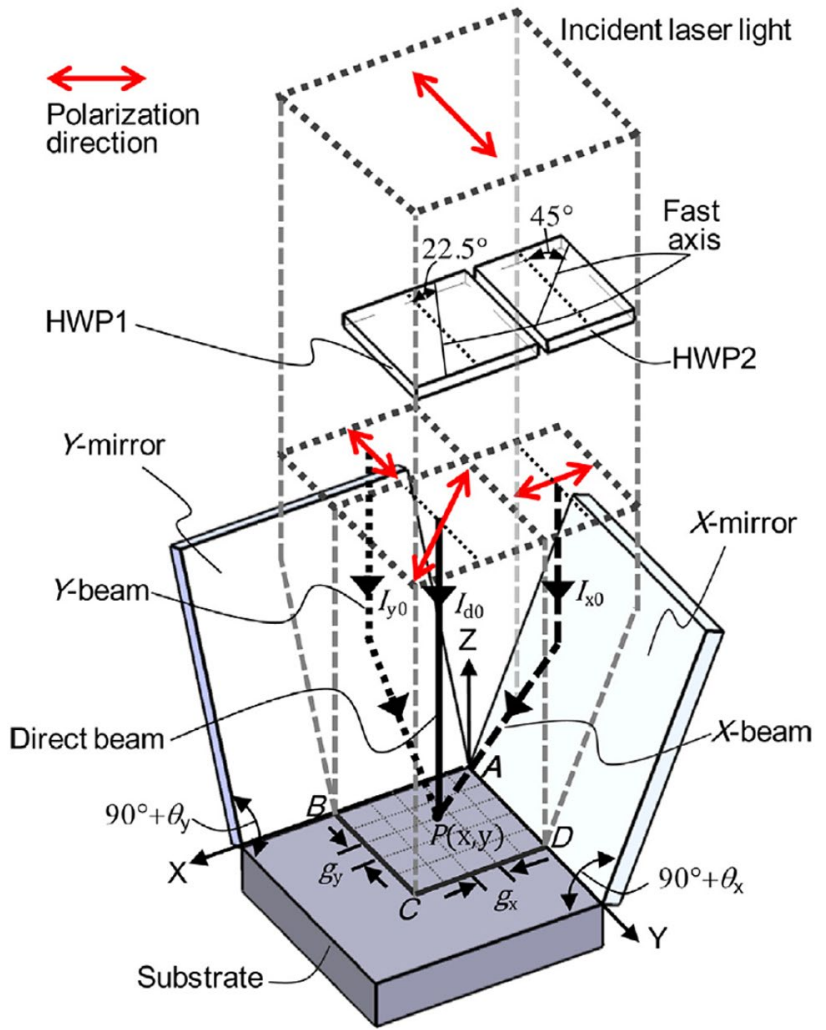

Fig. 16 A basic principle of the non-orthogonal two-axis Lloyd's mirror interferometer with a polarization modulation control [88]

control unit [88]. The interferometer is based on the division of wavefront method, and the main laser beam is divided into the following three sub-beams: 
Direct beam: Directly projected onto the substrate

$X$-beam: Enters the substrate after being reflected by the $X$-mirror

$Y$-beam: Enters the substrate after being reflected by the $Y$-mirror

The wavevectors $\overrightarrow{k_{1}}, \overrightarrow{k_{2}}, \overrightarrow{k_{3}}$ of the direct beam, $X$-beam and $Y$-beam, respectively, can be expressed by the following equation:

$\overrightarrow{k_{1}}=\frac{2 \pi}{\lambda}\left(\begin{array}{c}0 \\ 0 \\ -1\end{array}\right), \quad \overrightarrow{k_{2}}=\frac{2 \pi}{\lambda}\left(\begin{array}{c}\sin 2 \theta_{1 y} \\ 0 \\ -\cos 2 \theta_{1 y}\end{array}\right), \quad \overrightarrow{k_{3}}=\frac{\sqrt{2} \pi}{\lambda}\left(\begin{array}{c}0 \\ \sin 2 \theta_{2 x} \\ -\cos 2 \theta_{2 x}\end{array}\right)$

In the non-orthogonal two-axis Lloyd's mirror interferometer, periods $g_{X}^{\prime}$ and $g_{Y}^{\prime}$ of the interference fringe patterns to be generated by the interference between the direct beam and $X$-beam, the direct beam and $Y$-beam, respectively, can be expressed by the following equations [98, 99]:

$g_{X}^{\prime}=\frac{\lambda}{\sin 2 \theta_{1 y}}, \quad g_{Y}^{\prime}=\frac{\lambda}{\sin 2 \theta_{2 x}}$

The polarization direction of the main laser beam is aligned to be parallel with the $Y$-axis before being made incident to the polarization modulation control unit, which is composed of two half-wave plates. One of the halfwave plates is placed for the direct beam and its fast axis is adjusted to be $22.5^{\circ}$ with respect to the $Y$-axis. The other is placed for $X$-beam and its fast axis is adjusted to be $45^{\circ}$ with respect to the $Y$-axis. This optical configuration gives the $90^{\circ}$ difference of the polarization direction of $X$-beam with respect to $Y$-beam; namely, $X$-beam and $Y$-beam cannot interfere with each other, and the interference fringe component by the superposition of $X$-beam and $Y$-beam can vanish, as can be understood from Eq. (10). It should be noted that the fringe contrasts of the pair of the direct beam and $X$-beam, and that of the direct beam and $Y$-beam are reduced since the polarization difference between the beams is $45^{\circ}$ while the interference fringes by $X$-beam and $Y$-beam are eliminated. Figure 17 shows a schematic of the setup for the non-orthogonal two-axis Lloyd's mirror interferometer [88]. The main laser beam is generated from the laser beam from a $\mathrm{HeCd}$ laser source. Each of the $X$ - and $Y$-mirrors is mounted on a rotary stage to adjust the mirror angle with respect to the substrate. Figure 18 shows the AFM images of the two-dimensional grating pattern structures having a pitch of $0.57 \mu \mathrm{m}$ fabricated by the setup shown in Fig. 17 with and without the polarization modulation control [88]. As can be seen in the figure, the polarization modulation control was found to contribute to eliminating the influences of unnecessary interference fringe patterns by the interference between $X$-beam and $Y$-beam.

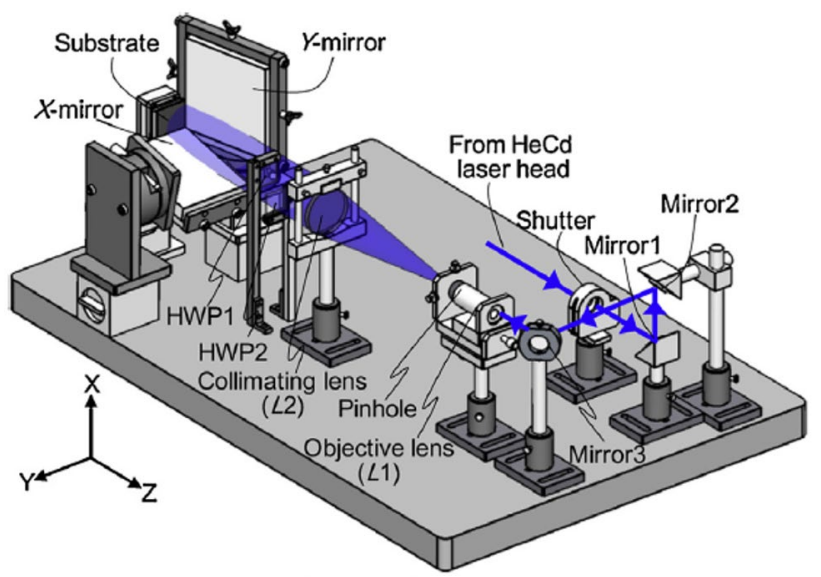

Fig. 17 A setup for the non-orthogonal two-axis Lloyd's mirror interferometer [88]

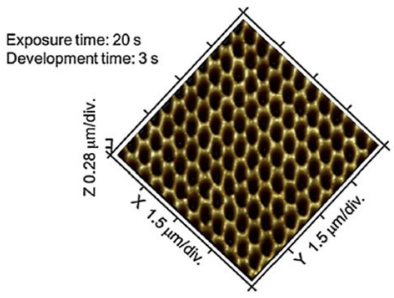

(a)

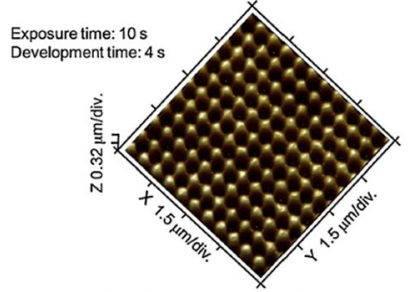

(b)
Fig. 18 AFM images of the orthogonal two-dimensional grating pattern structures fabricate by the non-orthogonal two-axis Lloyd's mirror interferometer [88]. a Without the polarization modulation control. b With the polarization modulation control

\subsubsection{Fabrication of a Large-Area Two-Dimensional Scale Grating}

In the non-orthogonal two-axis Lloyd's mirror interferometer, pattern exposure of a large-area two-dimensional scale grating can be carried out by employing large $X$ - and $Y$-mirrors and expanding the main laser beam to be made incident to the interferometer unit [98]. Figure 19a shows a setup for the fabrication of large-area two-dimensional scale grating [100]. The main laser beam can be expanded by employing a large-aperture lens for the collimation of the laser beam propagating from the spatial filter composed of a pinhole and an objective lens to construct a Keplerian beam expander. This optical configuration enables the interferometer to carry out the pattern exposure over a large area of $100 \mathrm{~mm} \times 100 \mathrm{~mm}$ [98]. However, the expansion of the main laser beam could reduce the light intensity on the substrate, and the pattern exposure time needs to be extended to satisfy the required exposure dose of the photoresist layer prepared on the substrate surface. However, the non-orthogonal two-axis Lloyd's mirror interferometer can accept the 
(a)

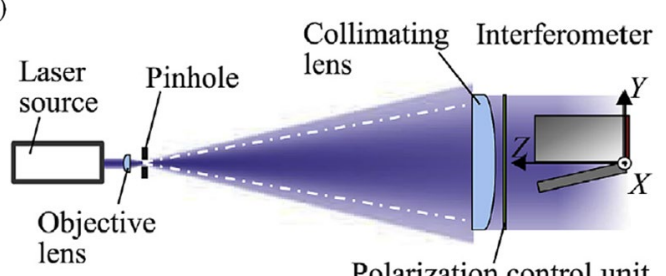

(b)

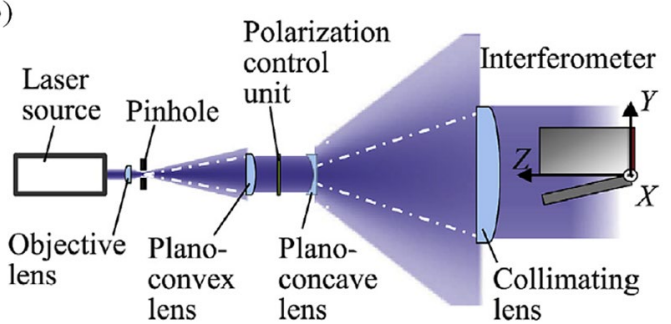

Fig. 19 Beam expansion units for the fabrication of a large-area scale grating [100]. a Based on the Keplerian beam expander. b Based on the Galilean beam expander

long exposure time since it is based on the division of wavefront method having high stability against external disturbances that come from the short non-common optical paths among the three sub-beams. It should be noted that a large polarization control unit is required for the setup shown in Fig. 19a. Meanwhile, it is not so easy to prepare for a large half-wave plate having a small retardance variation, resulting in incomplete polarization modulation control. This issue can be addressed by applying the Galilean beam expander in the optical setup as shown in Fig. 19b [100]. In the setup, the beam expansion is carried out in a two-step procedure. In the first step, the laser beam from the light source is made to pass through the spatial filter and is shaped in a collimated laser beam with a small diameter. The polarization modulation control is carried out at this stage by using the polarization control unit composed of two half-wave plates with a small retardance variation over the entire plate. After that, a pair of plano-concave lens and a large-aperture collimating lens is employed for the expansion of the laser beam after the polarization modulation control to obtain the main laser beam for the pattern exposure. Figure 20 shows the results of the numerical calculation for verifying the influences of imperfect polarization modulation control [100]. AFM images of the two-dimensional pattern structures fabricated by the setup without the polarization control, the setups with the optical configuration shown in Fig. 19a, b are also indicated. As can be seen in the figures, incomplete polarization control could result in distorted pattern structures. It is also revealed that the optical configuration shown in Fig. 19b is effective in removing the pattern distortion.

Another issue of the non-orthogonal two-axis Lloyd's mirror interferometer for the fabrication of a large-area scale grating is its light intensity distribution in the expanded main laser beam. In general, the laser beam passed through a spatial filter has a Gaussian light intensity distribution; namely, a large intensity difference can be found at the laser axis and the outer position in the expanded main laser beam. This issue can be neglected when a high-power laser source can be employed in the interferometer system. However, it is not so easy to operate such high-power laser sources in a laboratory where the available facilities and research budget are limited. The process optimization is thus required to achieve the fabrication of large-area scale grating having uniform pattern amplitude over a large area. In this case, not only the light intensity distribution of the two-dimensional interference fringe patterns but also the material property
Fig. 20 Influences of the imperfect polarization modulation control on the interference fringe patterns and the corresponding two-dimensional pattern structures to be obtained by the pattern exposure [100]. a Without the polarization control. b With the imperfect polarization modulation control. c With the improved polarization modulation control
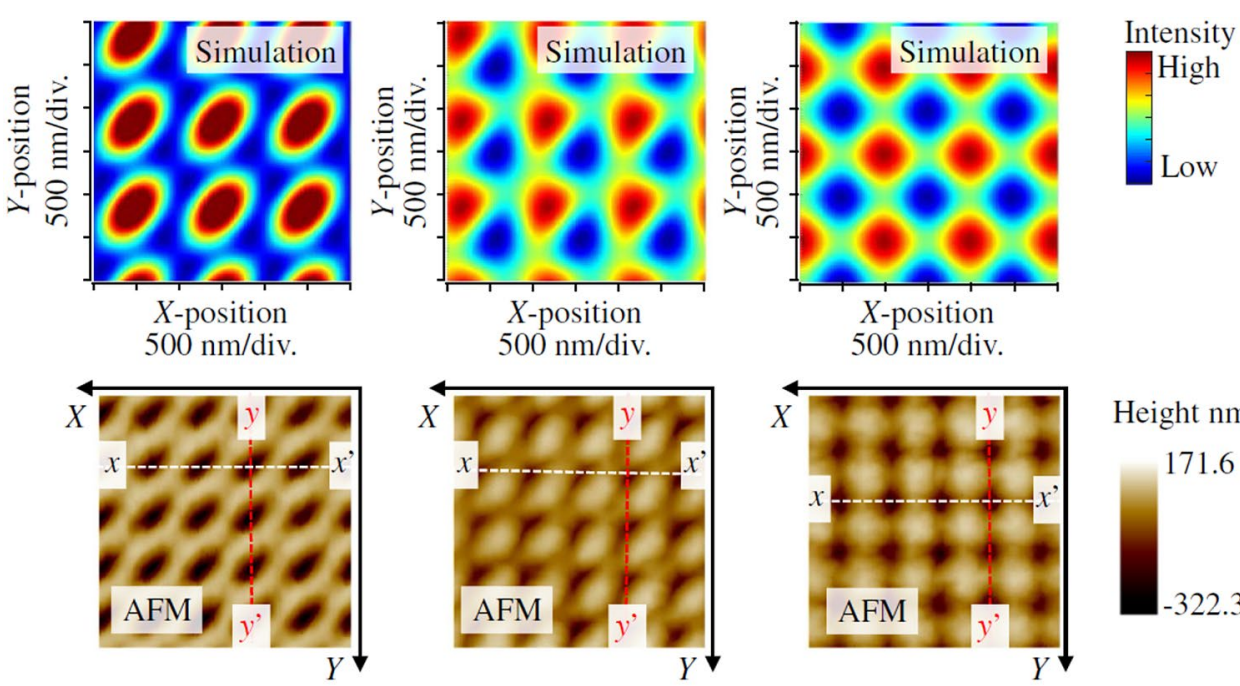

(a)

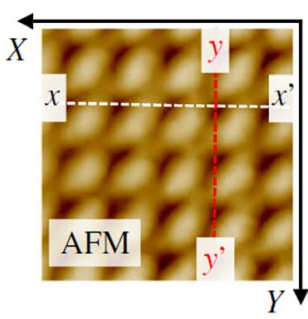

(b)

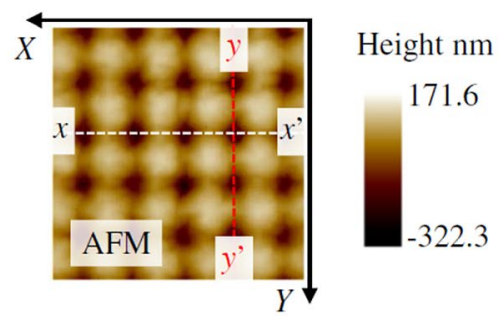

(c) 


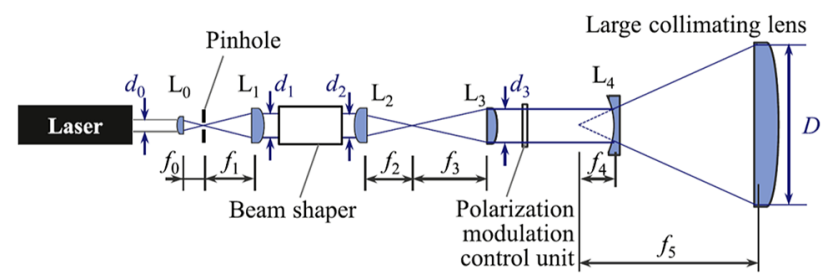

Fig. 21 A beam expansion unit with a beam shaper [101]

of the employed photoresist are required to be taken into consideration. One of the effective solutions for this issue is to employ a beam shaper composed of diffractive optical elements (DOEs) [101, 102]. Figure 21 shows an example of the optical setup employing a beam shaper [101]. In the setup, the main laser beam is expanded in a three-step procedure. At first, the laser beam from the laser source is made to pass through a spatial filter, and is shaped into a collimated laser beam with a small diameter. The collimated beam is then made incident to the beam shaper to obtain a laser beam having top-hat intensity distribution. After that, the polarization modulation control is carried out in the second step of the beam expansion, followed by the beam expansion by the pair of the plano-concave lens and a large-aperture collimating lens in the final step. Figure 22 compares the light intensity distribution of the main laser beams generated by the setup shown in Figs. 19a, b and 21 [101]. With the employment of the beam shaper, the main laser beam having high and uniform intensity over the entire region can be obtained; this contributes to fabricate two-dimensional grating pattern structures having uniform amplitude over a wide area.

Figure 23a, b show a schematic and a photograph of the non-orthogonal two-axis Lloyd's mirror interferometer equipped with the polarization modulation control unit and a beam shaper [101]. Figure 23c shows the two-dimensional scale grating in a size of $100 \mathrm{~mm} \times 100 \mathrm{~mm}$ fabricated through a single pattern exposure process. It should be noted that the scale grating shown in Fig. 23c has been fabricated in an ordinary laboratory room where strict environmental control has not been done; this fact means that the nonorthogonal Lloyd's mirror interferometer has high stability (a)
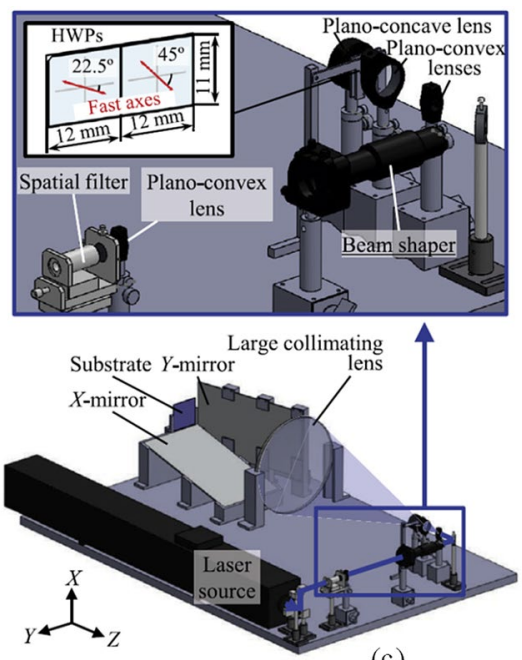

(b)

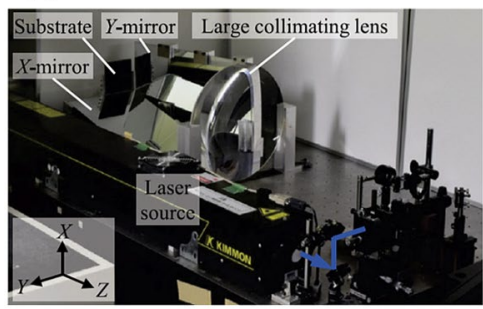

(c)

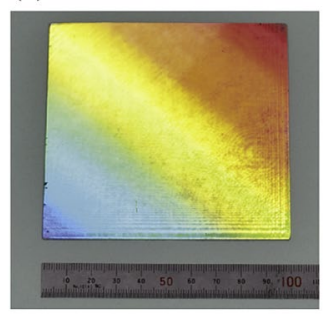

Fig. 23 A non-orthogonal two-axis Lloyd's mirror interferometer for the fabrication of a large-area two-dimensional scale grating [101]. a A schematic of the setup. b A photograph of the setup. c A twodimensional scale grating in a size of $100 \mathrm{~mm} \times 100 \mathrm{~mm}$ with a grating pitch of $1 \mu \mathrm{m}$

against the environmental fluctuations, and is suitable for the fabrication of large-area scale gratings.

\subsection{Current and Future Trends}

In this section, the multi-beam interference technology has been reviewed, while mainly focusing on the two types of two-axis Lloyd's mirror interferometers (the orthogonal-type and the non-orthogonal-type) for the fabrication of a twodimensional orthogonal diffraction grating to be employed as a scale in planar/surface encoders for multi-axis displacement measurement. Nowadays orthogonal two-dimensional

Fig. 22 Intensity distributions of the main laser beams generated by each of the beam expansion assembly [101]. a With the setup shown in Fig. 19a. b With the setup shown in Fig. 19b. c With the setup shown in Fig. 21 having a beam shaper (a)

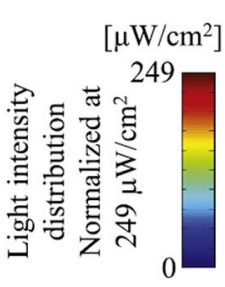

(b)

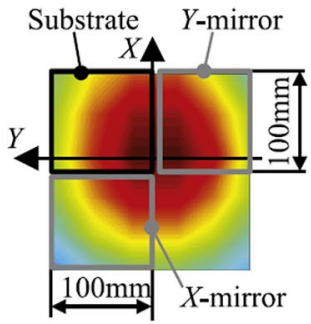

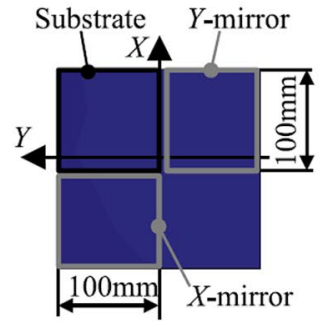

(c)

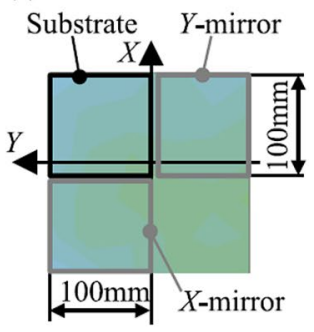




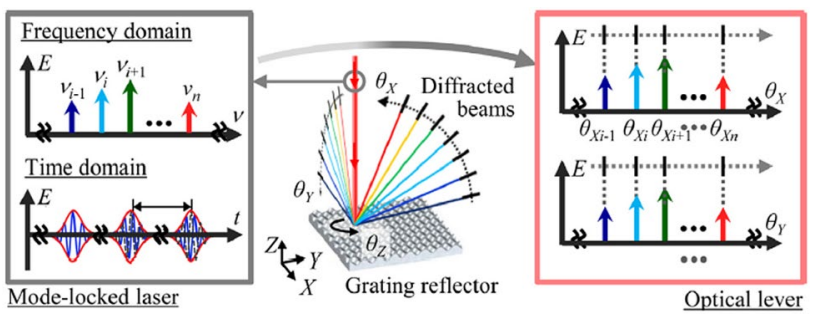

Fig. 24 An application of the two-dimensional diffraction grating in an optical angle sensor [103]

diffraction gratings are expanding their application in many industrial fields; for example, Fig. 24 shows an example of the applications in an optical angle sensor [103], where the two-dimensional diffraction grating is employed as a dispersive optical element. By combining a mode-locked femtosecond laser and a two-dimensional diffraction grating, highly accurate multi-axis measurement can be realized [104-107]. However, it is still difficult to find out appropriate two-dimensional diffraction gratings in commercial markets, and researchers/engineers should prepare for the two-dimensional diffraction gratings suitable for their applications by themselves; in such a case, the two-axis Lloyd's mirror interferometers reviewed in this paper could be a powerful solution for fabricating the grating by themselves for their purposes.

In the state-of-the-art scientific fields, studies on methods for fabricating three-dimensional fine structures such as photonic crystals [108-110], biomimetic structures [111], and nanoporous filters [112] have been actively carried out in recent years. One example is 3D lithography using the Talbot effect [112-114]. In this method, a periodic light intensity distribution is generated by using a diffraction grating, and is used for the photoresist exposure to fabricate threedimensional periodic fine structures in a large area. Various methods have been studied so far, including the method of using colloidal particles as a diffraction grating [115]. A method to increase the degree-of-freedom of designing the three-dimensional periodic structure has also been developed [116]. Another technological trend is the fabrication of narrower pattern structures. According to Eq. (3), the minimum pitch of the interference fringe patterns to be achieved by the interference lithography is a half of the light wavelength. The employment of a laser source having a shorter wavelength can reduce the pitch in the same manner as the technological trend that can be seen in the semiconductor industry [117]. Meanwhile, efforts have also been made to overcome the theoretical limitation by employing evanescent wave interference lithography [118-120]. Also, the in-situ monitoring of the pattern exposure process, which is being actively investigated in recent years, is expected to improve the accuracy of pattern structures to be obtained by the laser interference lithography $[121,122]$. The interference lithography is expected to play an important role in the fabrication of such functional optical elements, regarding the strong demands from the state-of-the-art scientific fields.

\section{Measurement Technology for the Evaluation of the Grating Pitch of a Diffraction Grating}

In some applications, the accuracy of a pattern pitch is not important; for example, the fabrication of functional surfaces for the improvement of tribological properties by applying surface textures [123]. On the other hand, in the optical multi-axis encoder system, the accuracy of the grating pitch directly affects the accuracy of displacement measurement $[7,8]$. The assurance of the grating pitch is thus one of the important issues to be addressed for achieving further higher positioning accuracy in a precision positioning system. Table 3 summarizes major methods for evaluating the pitch of a diffraction grating, which include critical-dimension scanning electron microscopes (SEMs) [42-46] or atomic force microscopes (AFMs) [47-52], vacuum interferometric comparators [124-128], laser diffractometric methods, and interferometric methods. In this section, these major methods for the evaluation of a grating pitch are reviewed.

\subsection{Critical-Dimension SEMs/AFMs (CD-SEMs, CD-AFMs)}

A direct and straightforward method to evaluate the grating pitch is to obtain the profile of grating pattern structures and detect the distance between the two neighboring pattern structures. Critical-dimension scanning electron microscopes (CD-SEMs) [42-46] and critical-dimension atomic force microscopes (CD-AFMs) [47-53] having a high lateral resolution can be employed for the purpose. Figure 25 shows an example of the critical-dimension AFM [53], where differential laser interferometers are employed to control the stage position. A grating pitch of less than $100 \mathrm{~nm}$ can be evaluated in a manner traceable to the national standard of length. However, in most of the cases, the in-plane measurement range of such measuring instruments with a high lateral resolution is limited (usually less than $100 \mu \mathrm{m} \times 100 \mu \mathrm{m}$ ). Many efforts have thus been made so far to expand the measurement range of such instruments with the enhancement of precision positioning systems, and nowadays a wide in-plane area over the several-hundred $\mathrm{mm}^{2}$ can be evaluated $[18$, $54,55]$. Meanwhile, it is still a difficult task to evaluate the whole area of a scale grating employed in an optical encoder, whose length can be longer than several-10 mm [129]. The expensive cost and long scanning time make it difficult to calibrate the whole area of a scale grating in a laboratorylevel condition. 
Table 3 Major methods for the evaluation of a grating pitch

Critical-dimension scanning electron microscopes (CD-SEMs)/atomic force microscopes (CDAFMs)

Vacuum interferometric comparators

Laser diffractometric methods

Interferometric methods
Direct and straightforward pitch evaluation from the obtained scale grating profile

A high lateral resolution better than sub-nm

Pattern structures with a sub-micrometric pitch can be evaluated

Absolute grating pitch can be obtained

The evaluation of a long scale grating is not a realistic task

A high instrumentation cost

Detect edges of a grating pattern structure for pitch measurement

Long scale gratings can be evaluated

Scale grating with a micrometric pitch can be evaluated

Lateral resolution can be improved with the employment of a short-wavelength light source in the edge sensor

Strict environmental control is required for the laser interferometers

A high instrumentation cost

Grating pitch deviation can be evaluated through detecting the change in the angle of diffraction of diffracted laser beams from a grating under evaluation

Long scale grating can be evaluated

Instrumentation is possible even in a small laboratory

Only the "mean" grating pitch in the area irradiated by the measurement laser beam can be obtained

A high-precision rotary stage is required (depending on the setup)

A rapid evaluation of the pitch deviation is possible

Large-scale grating can be evaluated with the enhancement of a large FOV of an interferometer
Fig. 25 A critical-dimension atomic force microscope $(\mathrm{CD}$ AFM) for the evaluation of a nanometric lateral scales [53]

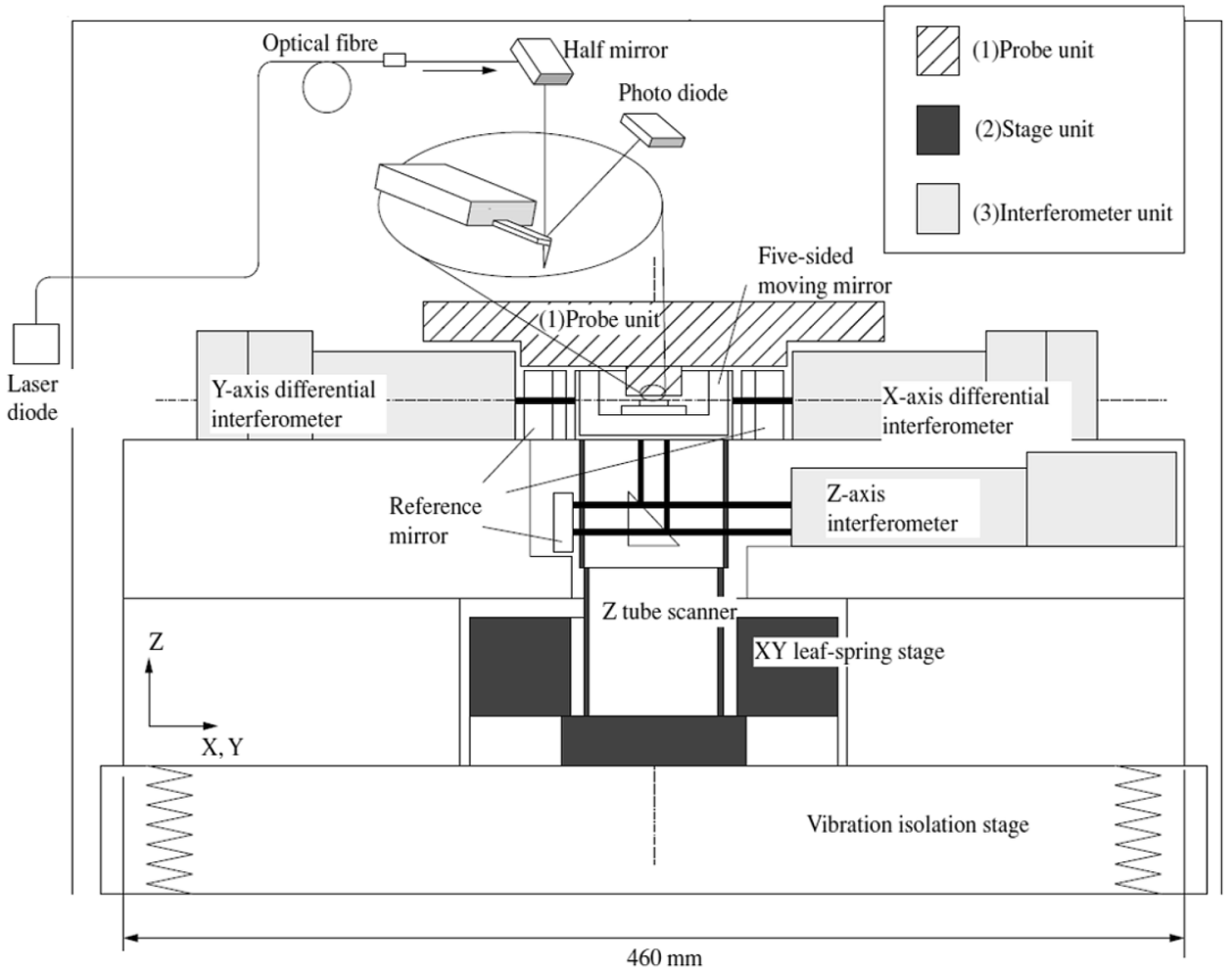




\subsection{Vacuum Interferometric Comparator}

Vacuum interferometric comparators can be employed to evaluate the grating pitch of a scale grating over its whole length [124-128]. Figure 26 shows a schematic of the vacuum interferometric comparator developed for the calibration of a linear scale for linear encoders [125]. In the setup, interferometers are fully arranged in a vacuum, while a scale grating to be evaluated is placed in the atmospheric condition. By making an optical head scan over the linear scale while measuring the scanning position by the laser interferometer, the pitch deviation of a scale grating can be calibrated. Figure 26b shows a schematic of the principle of edge detection by the optical head in the comparator. The light intensities of transparent light through the three slits captured by photodetectors are employed to detect the edge of grating pattern structures through signal processing. Since the position of the detected edge of a grating pattern structure can be assured by the reading of the laser interferometer, a grating pitch can be evaluated by detecting the edges of each of the grating pattern structures. A long linear scale grating with a length of $1600 \mathrm{~mm}$ can be successfully calibrated with a resolution of $0.8 \mathrm{~nm}$ [125]. The concept of the comparator can also be expanded to the evaluation of two-dimensional pattern structures [130]. Meanwhile, these setups require facilities with a high degree of environmental control. A high cost for the instrumentation is also an obstacle to constructing a comparator in ordinary research laboratories where research budgets and available facilities are limited.

\subsection{Laser Diffractometry}

Figure 27a shows a setup for measurement of the grating pitch by using the diffracted beam [131]. In the setup, a grating under measurement is mounted on a high-precision rotary table equipped with a high-precision angle sensor such as an optical rotary encoder. The variation of a grating pitch affects the angle of diffraction of diffracted beams from a diffraction grating. According to the grating equation [62], in the case of the normal incidence, the following relationship can be found among the light wavelength $\lambda$, grating pitch $g$ and the angle of diffraction $\beta_{m}$ of the $m$ th-order diffracted beam:

$\sin \beta_{m}=\frac{\lambda}{g}$

On the assumption that $\lambda$ is known, it is possible to evaluate $g$ by detecting the angle of diffraction of diffracted beams [131-134]. By utilizing the Littrow configuration, where the reflected laser beam propagates in the opposite direction of the propagation of the incident laser beam, $\beta_{m}$ can easily be obtained. Although this method cannot directly evaluate the grating pitch as CD-SEMs or CD-AFMs do, they can carry out fast pitch measurement over a wide measuring area. It has also been reported that a good agreement can be found between the results obtained by a CD-AFM and an optical setup based on the laser diffraction method [135]; this fact means that the laser diffraction method can carry out reliable measurement of a grating pitch. The accuracy of the pitch measurement by the laser diffraction method can be improved by employing several diffracted beams [133]. Furthermore, when a mode-locked femtosecond laser beam
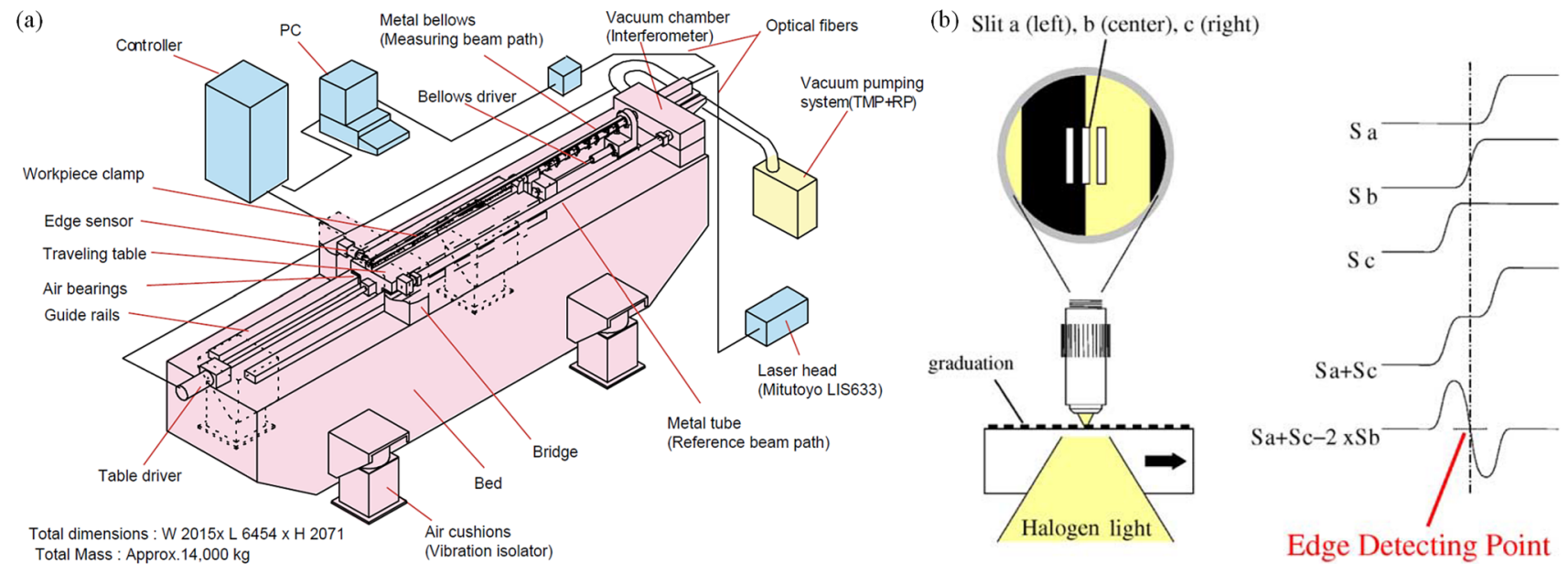

Total Mass : Approx. $14,000 \mathrm{~kg}$

Fig. 26 Vacuum interferometric comparator for the calibration of a linear scale for linear encoders [125]. a A schematic of the whole system setup of the comparator. b A principle of detecting the edge of a grating pattern structure 
(a)

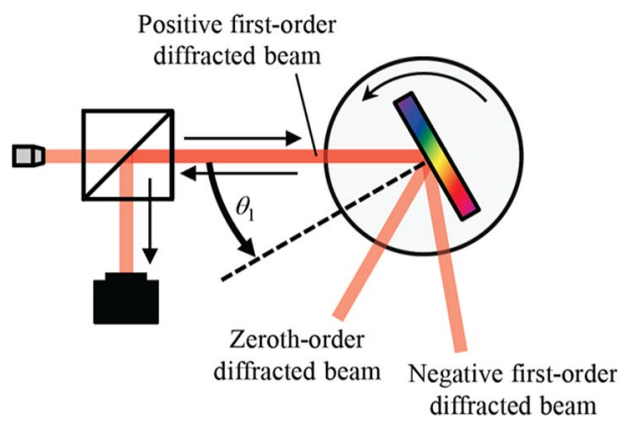

(b)

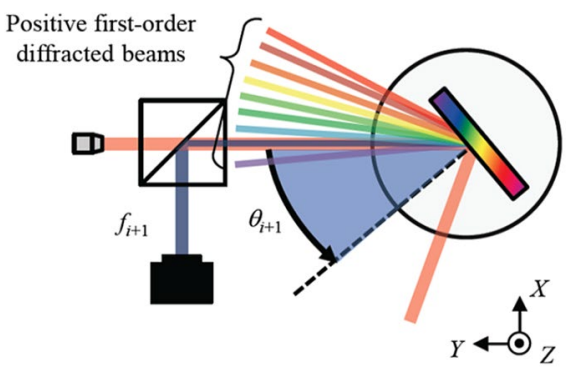

Fig. 27 The evaluation of a grating pitch based on the laser diffraction method [131]. a Measurement of the positive first-order diffracted beam in the Littrow configuration. b The laser diffraction method utilizing multiple first-order diffracted beams generated by a mode-locked femtosecond laser source having multiple optical modes

is employed as the light source for the grating pitch measurement, many diffracted beams in the same diffraction order from the same grating area can be obtained as shown in Fig. 27b; by detecting the angles of diffraction of these diffracted beams, highly accurate measurement of the pitch deviation can be achieved [131].

By scanning the measurement laser beam over a surface of the scale grating under measurement in the laser diffraction method, a fast evaluation of the pitch deviation of a scale grating can be achieved [59, 136, 137]. Figure 28 shows an example of the optical configurations for the evaluation of grating pitch deviation. In the setup, a grating under measurement is initially placed to satisfy the Littrow configuration where the diffracted beam propagates in the opposite direction to the incident laser beam. After that, an optical head is made to scan over the grating under measurement. Due to the pitch deviation of the grating, the diffracted beam experiences the change in the angle of diffraction that can be detected by optical methods such as the long trace profiler (LTP) [136, 138] or the laser autocollimation [59, 139]. The LTP system is based on the pencil beam interferometer [140] that employs a pair of parallel and separated narrow beams (referred to as the pencil beams). A pair of the diffracted pencil beams are focused on a photodetector by a Fouriertransform lens, and generate an interference fringe pattern. Since the change in the angles of the diffracted pencil beams is converted into the displacement of the interference fringe

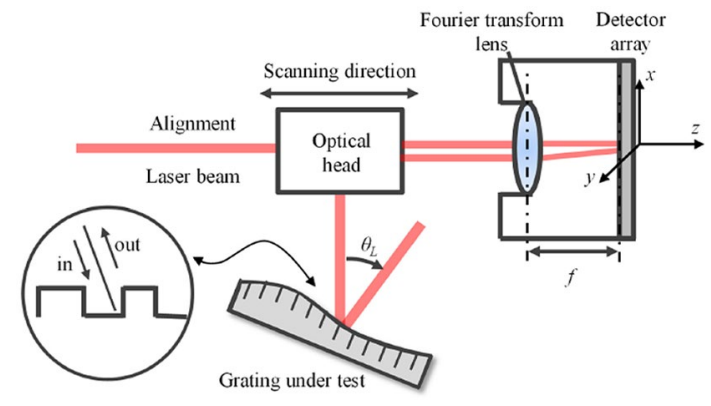

Fig. 28 The evaluation of a grating pitch deviation based on the laser diffraction method with a long trace profiler (LTP)

pattern on the photodetector, the grating pitch deviation can be detected by observing the position of the interference fringe pattern on the photodetector. By employing a pentaprism for the scanning of the laser beam, the influence of the angular error motion of the scanning can be reduced [136]. Furthermore, by detecting both the zeroth-order diffracted beams and the first-order diffracted beam, the grating pitch can be evaluated while reducing the influence of the local slope of a grating surface [137]. One of the drawbacks of the setup shown in Fig. 28 is its low resolution for measurement of the angle of diffraction of the laser beam. This can be overcome by the optical configuration based on the laser autocollimation [59, 139]. With the employment of photodiodes (PDs) as the photodetectors for the detection of the diffracted beams, a high-resolution measurement can be realized while designing the optical head in a compact size [141-144]. Figure 29a shows one of the examples of the optical configuration for the laser diffraction method based on the laser autocollimation [59, 145, 146]. The setup is designed to capture the zeroth and first-order diffracted beams from the scale grating under measurement. Through the arithmetic processing with the two readings out of these three diffracted beams, the grating pitch deviation of a scale grating can be evaluated without being affected by the angular error motion of the scanning of the scale grating. Denoting the angles of diffraction of the positive and negative first-order diffracted beams as $\theta_{\text {Pos }}(x)$ and $\theta_{\mathrm{Neg}}(x)$, respectively, the pitch deviation $\Delta g$ can be obtained by the following equation [59]:

$\Delta g \cong \frac{\lambda}{\frac{\lambda}{\bar{g}}+\frac{\theta_{\mathrm{Pos}}(x)-\theta_{\mathrm{Neg}}(x)}{2}}-\bar{g}$

where $g$ is the nominal grating pitch. It should be noted that the influences of the local slope of the scale and the angular error motion of the scale scanning are cancelled in the above equation; namely, the setup shown in Fig. 29a can carry out grating pitch deviation measurement in a stable manner. Also, through the arithmetic processing of the readings 
(a)

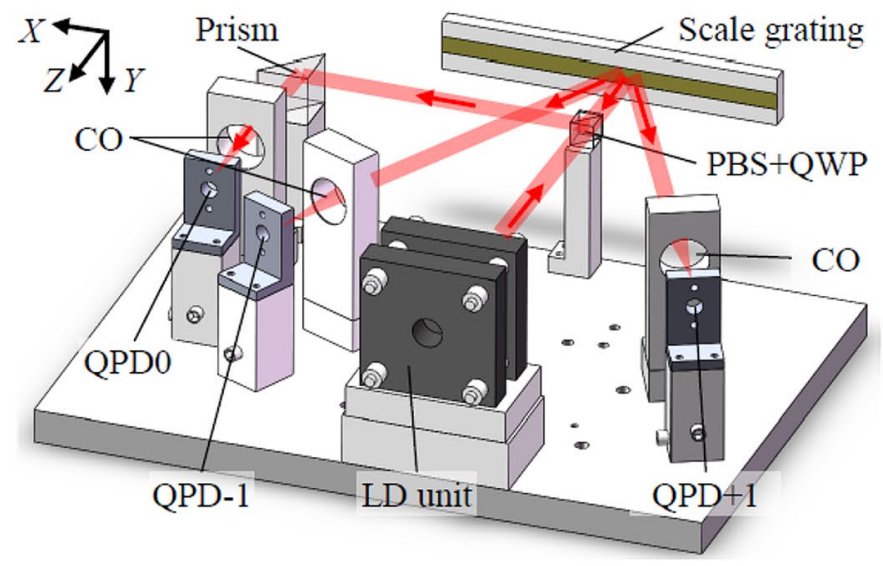

(b)

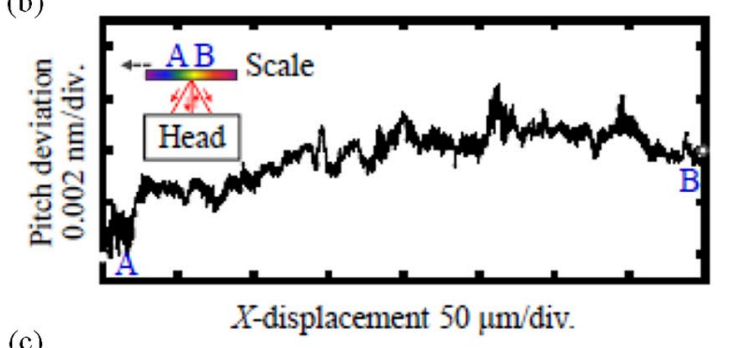

(c)

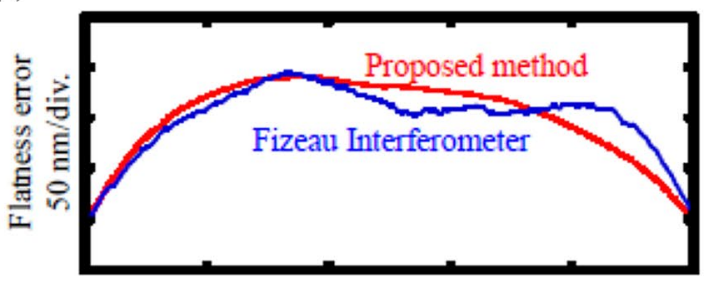

$X$-position $20 \mathrm{~mm} /$ div.
Fig. 29 The evaluation of a grating pitch deviation based on the laser diffraction method with a multi-axis laser autocollimator [59]. a Optical sensor head. b Pitch deviation of a scale grating from a commer-

of the angle sensor, the local slope of the scale grating $\Delta \theta_{\text {surface }}(x)$ can be obtained as follows [59]:

$\Delta \theta_{\text {surface }}(x)=-\frac{\left(\theta_{\mathrm{Pos}}^{\prime}(x)+\theta_{\mathrm{Neg}}(x)\right)}{4}-\Delta \theta_{\text {stage }}(x)$

where $\Delta \theta_{\text {stage }}(x)$ is the angular error motion of the scale scanning. Figure $29 \mathrm{~b}$ shows the pitch deviation of a scale grating from a commercial interferential scanning-type optical linear encoder measured by the setup shown in Fig. 29a [59]. As can be seen in the figure, a high resolution of better than $0.002 \mathrm{~nm}$ can be obtained. Furthermore, with the enhancement of the additional angle sensor for measurement of the angular error motion of the scale scanning $\left(\Delta \theta_{\text {stage }}(x)\right)$, the flatness error of the scale grating can also be evaluated by using the readings of the optical head based on the laser autocollimation. Figure 29c shows the flatness error of a scale grating measured by the setup shown in Fig. 29a [59]. As can be seen in the figure, a good agreement can be found between the obtained flatness error and the grating profile measured by a commercial Fizeau interferometer [61].

\subsection{Laser Interferometric Method}

In the above-mentioned methods, a two-dimensional scale grating needs to be moved by using a two-dimensional positioning system for the evaluation of the grating pitch along the $X$ - and $Y$-directions. Meanwhile, in the interferometric method where the grating pitch deviation is detected as the phase shift in the wavefront of the diffracted beams, a largearea scale grating can be evaluated in a short time $[60,61$, 147]. Figure 30a shows a schematic of how the grating pitch cial optical linear encoder measured by the setup $\mathbf{c}$ flatness error of the measured scale grating reconstructed from the sensor reading

deviation could affect the phase in the wavefront of the diffracted beam [61]. The wavefront of a first-order diffracted beam can be evaluated by using a Fizeau interferometer; place the grating in the Littrow configuration under the measurement laser beam of the interferometer as shown in Fig. 30b. For the evaluation of the $X$ - and $Y$-directional pitch deviations of two-dimensional grating pattern structures, the scale grating needs to be rotated about the $Y$ - and the $X$-axes, respectively, to obtain the wavefronts of the diffracted beams.

Attention should also be paid to the flatness error of the scale grating when evaluating the grating pitch deviation based on the laser interferometric method, since the out-offlatness error could also affect the wavefront of the diffracted beam. This issue can be addressed by the arithmetic operation with the wavefronts of the positive and negative firstorder diffracted beams. Now the wavefronts of the $X$-directional positive and negative first-order diffracted beams, $I_{X+1}$ and $I_{X-1}$, respectively, can be expressed as follows [61]:

$I_{X+1}(x, y)=2 \pi \frac{e_{X}(x, y)}{g}+2 \pi \frac{2 e_{Z}(x, y)}{\lambda} \cos \frac{\theta_{\text {Littrow }}}{2}$

$I_{X-1}(x, y)=-2 \pi \frac{e_{X}(x, y)}{g}+2 \pi \frac{2 e_{Z}(x, y)}{\lambda} \cos \frac{\theta_{\text {Littrow }}}{2}$

where $e_{X}(x, y)$ and $e_{Z}(x, y)$ denote the $X$-directional pitch deviation and the out-of-flatness error of the scale grating, and $\theta_{\text {Littrow }}$ is the Littrow angle. From these two equations, the $X$-directional pitch deviation $e_{X}(x, y)$ can be obtained as follows while cancelling the out-of-flatness error $e_{Z}(x, y)$, and vice versa [61]: 
(a)

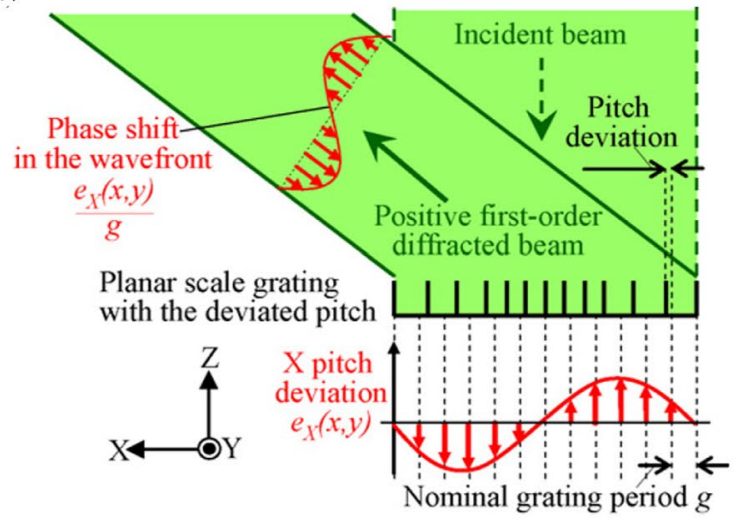

Fig. 30 The evaluation of a grating pitch deviation based on the laser interferometric method with a Fizeau interferometer [61]. a A phase shift in the wavefront of the diffracted beam due to the deviated grat-

$e_{X}(x, y)=\frac{g}{4 \pi}\left[I_{X+1}(x, y)-I_{X-1}(x, y)\right]$

In the same manner, the $Y$-directional pitch deviation $e_{Y}(x, y)$ can be obtained from the $Y$-directional positive and negative first-order diffracted beams. Figure 31a-c show the grating pitch deviation and the out-of-flatness error of a two-dimensional scale grating measured by the laser interferometric method with a commercial Fizeau interferometer. Arithmetic operations described by Eqs. (16), (17), and (18) are applied to obtain these results. The two-dimensional grating fabricated by the laser interference lithography with a nominal grating pitch of $1 \mu \mathrm{m}$ has successfully been evaluated. This method can carry out a fast evaluation of the twodimensional diffraction grating over the area within the fieldof-view (FOV) of the Fizeau interferometer. The validity of the above method has been confirmed by the good agreement of the measured pitch deviation and the out-of-flatness (b)

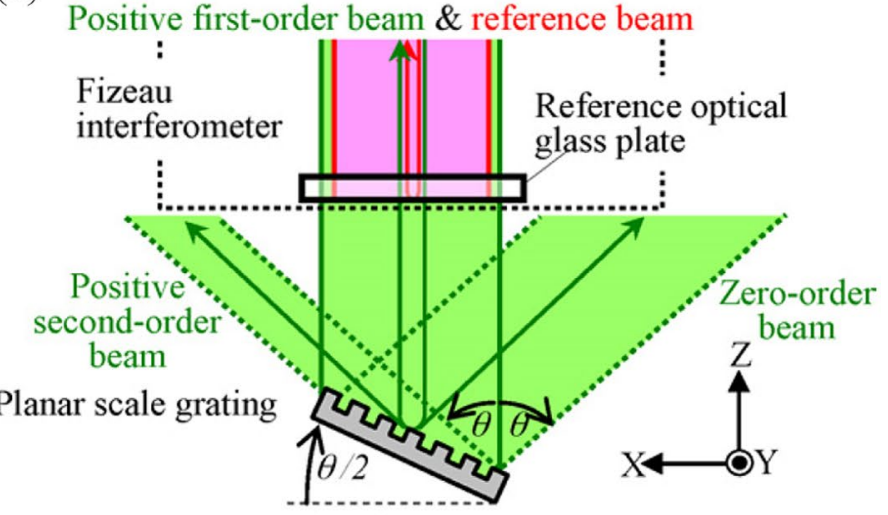

ing pitch. b Measurement of the wavefront of positive first-order diffracted beam under the Littrow configuration

error of the scale grating with the errors in the readings of the three-axis surface encoder [61].

It should be noted that attention should be paid for the out-of-flatness of the reference optical flatness in the Fizeau interferometer, since it usually has a peak-to-valley (PV) value of $\lambda / 20$, and cannot be neglected in the precision evaluation of the grating pitch. This issue can be addressed by employing the wavefronts of the positive and negative firstorder diffracted beams, as well as the zeroth-order diffracted beam, in the modified arithmetic operations [148-150]; namely, evaluation of the pitch deviation of a scale grating can be carried out while self-calibrating the Fizeau interferometer employed in the pitch evaluation. (a)

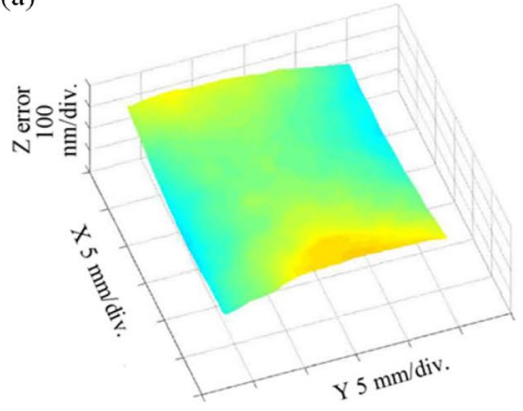

(b)

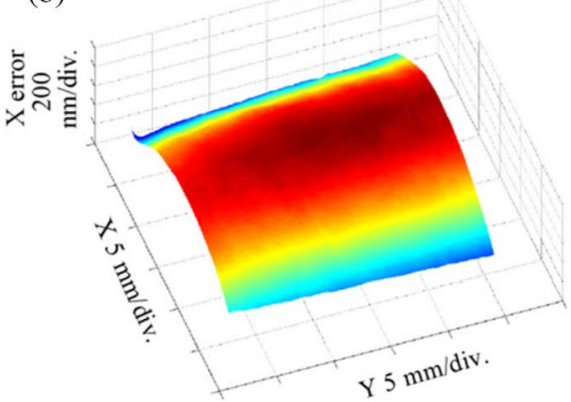

(c)

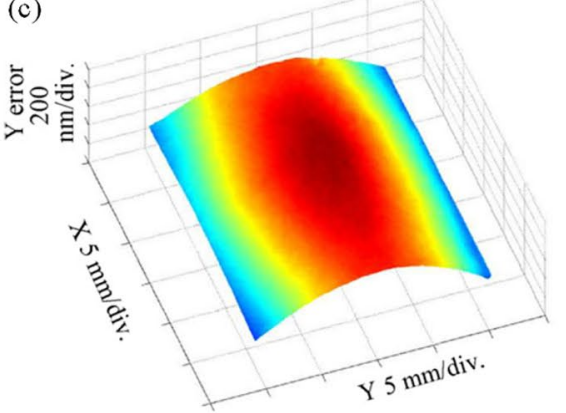

Fig. 31 Grating pitch deviation and the out-of-flatness error of a two-dimensional scale grating measured by the laser interferometric method with a commercial Fizeau interferometer [61]. The twodimensional grating has been fabricated by the laser interference lithography, and has a nominal grating pitch of $1 \mu \mathrm{m}$. a Out-of-flatness of the scale grating. b $X$-directional pitch deviation. c $Y$-directional pitch deviation 


\section{Conclusions}

In this paper, some techniques for the fabrication of a diffraction grating based on the laser interference lithography have been reviewed, while focusing on the fabrication of orthogonal two-dimensional gratings to be employed as a scale for multi-axis planar/surface encoder. Lloyd's mirror interferometers, which are based on the division of wavefront system, have superior stability against the external disturbances, and are thus suitable for the fabrication of a large-area two-dimensional scale grating that requires long pattern exposure time. Some innovations have been made to the conventional one-axis Lloyd's mirror interferometer to realize the fabrication of a high-precision two-dimensional scale grating at a single exposure. Optimizations of the optical setup have also been carried out to fabricate a large-area two-dimensional scale grating required in the state-of-the-art scientific and industrial fields.

Some major methods for measurement of a grating pitch have also been reviewed in this paper since the accuracy of the grating pitch is one of the important aspects of the diffraction grating, especially when it is employed as the scale for displacement measurement in an optical encoder system. A diffraction grating is one of the most important optical components in many scientific fields, and many efforts have thus been made so far to evaluate the grating pitch. Among them, optical techniques based on the laser diffraction method and the laser interferometric method are appropriate ones for the evaluation of a scale grating to be employed in optical encoder systems, regarding the required large measurement range, and even now some research projects are being actively carried out.

Since it is still difficult to find out commercially available two-dimensional diffraction gratings in a market, the establishments of fabrication and evaluation technologies of a two-dimensional scale grating described in this paper allow researcher/engineers to fabricate their gratings appropriate for their applications even in their ordinary research laboratories where available facilities and research budgets are limited. These technologies are expected to accelerate the research activities where a large-area two-dimensional diffraction grating plays an important role as a scale for multiaxis displacement or a diffractive optical element in optical measuring instruments and products.

Acknowledgements A part of the research activities described in this paper is supported by the Japan Society for the Promotion of Science (JSPS). The author would like to thank Professor Wei Gao (Tohoku University, Japan) for the fruitful discussions and valuable comments on the topic of this review article.

\section{Compliance with Ethical Standards}

Conflict of interest The corresponding author states that there is no conflict of interest.

Open Access This article is licensed under a Creative Commons Attribution 4.0 International License, which permits use, sharing, adaptation, distribution and reproduction in any medium or format, as long as you give appropriate credit to the original author(s) and the source, provide a link to the Creative Commons licence, and indicate if changes were made. The images or other third party material in this article are included in the article's Creative Commons licence, unless indicated otherwise in a credit line to the material. If material is not included in the article's Creative Commons licence and your intended use is not permitted by statutory regulation or exceeds the permitted use, you will need to obtain permission directly from the copyright holder. To view a copy of this licence, visit http://creativecommons.org/licenses/by/4.0/.

\section{References}

1. Kneubühl F (1969) Diffraction grating spectroscopy. Appl Opt 8:505

2. Shank CV, Fork RL, Yen R, Stolen RH, Tomlinson WJ (1982) Compression of femtosecond optical pulses. Appl Phys Lett 40:761-763

3. Canova F, Clady R, Chambaret J-P, Flury M, Tonchev S, Fechner R, Parriaux O (2007) High-efficiency, broad band, high-damage threshold high-index gratings for femtosecond pulse compression. Opt Express 15:15324

4. Treacy E (1969) Optical pulse compression with diffraction gratings. IEEE J Quantum Electron 5:454-458

5. Draper CT, Bigler CM, Mann MS, Sarma K, Blanche P-A (2019) Holographic waveguide head-up display with 2-D pupil expansion and longitudinal image magnification. Appl Opt 58:A251

6. Suhara T, Nishihara H (1986) Integrated optics components and devices using periodic structures. IEEE J Quantum Electron 22:845-867

7. Gao W, Kim SW, Bosse H, Haitjema H, Chen YL, Lu XD, Knapp W, Weckenmann A, Estler WT, Kunzmann H (2015) Measurement technologies for precision positioning. CIRP Ann Manuf Technol 64:773-796

8. Teimel A (1992) Technology and applications of grating interferometers in high-precision measurement. Precis Eng 14:147-154

9. Li X, Gao W, Muto H, Shimizu Y, Ito S, Dian S (2013) A sixdegree-of-freedom surface encoder for precision positioning of a planar motion stage. Precis Eng 37:771-781

10. Shimizu Y, Matsukuma H, Gao W (2019) Optical sensors for multi-axis angle and displacement measurement using grating reflectors. Sensors 19:5289

11. Gao W, Kimura A (2007) A Three-axis displacement sensor with nanometric resolution. CIRP Ann Manuf Technol 56:529-532

12. Kimura A, Gao W, Kim W, Hosono K, Shimizu Y, Shi L, Zeng L (2012) A sub-nanometric three-axis surface encoder with shortperiod planar gratings for stage motion measurement. Precis Eng 36:576-585

13. Lin C, Yan S, Ding D, Wang G (2018) Two-dimensional diagonal-based heterodyne grating interferometer with enhanced signal-to-noise ratio and optical subdivision. Opt Eng 57:064102

14. Hsieh H-L, Pan S-W (2015) Development of a grating-based interferometer for six-degree-of-freedom displacement and angle measurements. Opt Express 23:2451

15. Heidenhain (2017) Encoders for machine tool inspection and acceptance testing. www.heidenhain.de 
16. Gao W, Dejima S, Shimizu Y, Kiyono S (2003) Precision measurement of two-axis positions and tilt motions using a surface encoder. CIRP Ann Manuf Technol 52:435-438

17. Matsukuma H, Ishizuka R, Furuta M, Li X, Shimizu Y, Gao W (2019) Reduction in Cross-talk errors in a six-degree-of-freedom surface encoder. Nanomanuf Metrol 2:111-123

18. Manske E, Jäger G, Hausotte T, Fül R, Füßl R (2012) Recent developments and challenges of nanopositioning and nanomeasuring technology. Meas Sci Technol 23:074001

19. Fan KC, Fei YT, Yu XF, Chen YJ, Wang WL, Chen F, Liu YS (2006) Development of a low-cost micro-CMM for 3D micro/ nano measurements. Meas Sci Technol 17:524-532

20. Ito S, Kikuchi H, Chen Y, Shimizu Y, Gao W, Takahashi K, Kanayama T, Arakawa K, Hayashi A (2016) A micro-coordinate measurement machine (CMM) for large-scale dimensional measurement of micro-slits. Appl Sci 6:156

21. Ito S, Kodama I, Gao W (2014) Development of a probing system for a micro-coordinate measuring machine by utilizing shearforce detection. Meas Sci Technol 25:064011

22. Butler H (2011) Position control in lithographic equipment: an enabler for current-day chip manufacturing. IEEE Control Syst 31:28-47

23. Shimizu Y, Ito T, Li X, Kim W, Gao W (2014) Design and testing of a four-probe optical sensor head for three-axis surface encoder with a mosaic scale grating. Meas Sci Technol 25:094002

24. Kimura A, Hosono K, Kim WJ, Shimizu Y, Gao W, Zeng L (2011) A two-degree-of-freedom linear encoder with a mosaic scale grating. Int J Nanomanuf 7:73-91

25. Hosono K, Kim WJ, Kimura A, Shimizu Y, Gao W (2011) Surface encoders for a mosaic scale grating. Int J Autom Technol 5:91-96

26. Harrison GR (1949a) The production of diffraction gratings I development of the ruling art*. J Opt Soc Am 39:413

27. Harrison GR (1949b) The production of diffraction gratings: II the design of Echelle gratings and spectrographs1. J Opt Soc Am 39:522

28. Evans S (1981) Design and construction of a large grating ruling engine. Precis Eng 3:193-200

29. Konkola PT, Chen CG, Heilmann RK, Joo C, Montoya JC, Chang C-HH, Schattenburg ML (2003) Nanometer-level repeatable metrology using the nanoruler. J Vac Sci Technol B Microelectron Nanom Struct 21:3097-3101

30. Gao W, Haitjema H, Fang FZ, Leach RK, Cheung CF, Savio E, Linares JM (2019) On-machine and in-process surface metrology for precision manufacturing. CIRP Ann 68:843-866

31. Fang FZ, Zhang XD, Gao W, Guo YB, Byrne G, Hansen HN (2017) Nanomanufacturing - perspective and applications. CIRP Ann Manuf Technol 66:683-705

32. Gao W, Araki T, Kiyono S, Okazaki Y, Yamanaka M (2003) Precision nano-fabrication and evaluation of a large area sinusoidal grid surface for a surface encoder. Precis Eng 27:289-298

33. Gao W, Chen YL, Lee KW, Noh YJ, Shimizu Y, Ito S (2013) Precision tool setting for fabrication of a microstructure array. CIRP Ann Manuf Technol 62:523-526

34. Stuerzebecher L, Fuchs F, Harzendorf T, Zeitner UD (2014) Pulse compression grating fabrication by diffractive proximity photolithography. Opt Lett 39:1042

35. Rogers JA, Paul KE, Jackman RJ, Whitesides GM (1997) Using an elastomeric phase mask for sub-100 $\mathrm{nm}$ photolithography in the optical near field. Appl Phys Lett 70:2658-2660

36. Lindau S (1982) The groove profile formation of holographic gratings. Opt Acta (Lond) 29:1371-1381

37. Brueck SRJ (2005) Optical and interferometric lithographynanotechnology enablers. Proc IEEE 93:1704-1721
38. Lu C, Lipson RH (2010) Interference lithography: a powerful tool for fabricating periodic structures. Laser Photonics Rev 4:568-580

39. Moon JH, Ford J, Yang S (2006) Fabricating three-dimensional polymeric photonic structures by multi-beam interference lithography. Polym Adv Technol 17:83-93

40. Burrowg GM, Gaylord TK (2011) Multi-beam interference advances and applications: Nano-electronics, photonic crystals, metamaterials, subwavelength structures, optical trapping, and biomedical structures. Micromachines 2:221-257

41. Chen Y-L, Shimizu Y, Tamada J, Nakamura K, Matsukuma H, Chen X, Gao W (2018) Laser autocollimation based on an optical frequency comb for absolute angular position measurement. Precis Eng 54:284-293

42. Nakayama Y, Gonda S, Misumi I, Kurosawa T, Kitta J, Mine H, Sasada K, Yoneda S, Mizuno T (2005) Novel CD-SEM calibration reference patterned by EB cell projection lithography. In: Silver RM (ed) Metrology, inspection, and process control for microlithography, vol 19. SPIE, Washington, p 591

43. Villarrubia JS, Vladar AE, Postek MT (2003) Simulation study of repeatability and bias in the CD-SEM. In: Herr DJ (ed) Metrology, inspection, and process control for microlithography, vol 17. SPIE, Washington, $\mathrm{p} 138$

44. Takamasu K, Kuwabara K, Takahashi S, Mizuno T, Kawada H (2010) Sub-nanometer calibration of CD-SEM line width by using STEM. In: Raymond CJ (ed) Metrology, inspection and process control for microlithography, vol 24. SPIE, Washington, $\mathrm{p} 76381 \mathrm{~K}$

45. Nakayama Y, Yamamoto J, Kawada H (2009) Sub-50-nm pitch size grating reference for CD-SEM magnification calibration. In: Allgair JA, Raymond CJ (eds) Metrology, inspection, and process control for microlithography, vol 23. SPIE, Washington, $\mathrm{p} 727224$

46. Orji NG, Badaroglu M, Barnes BM, Beitia C, Bunday BD, Celano U, Kline RJ, Neisser M, Obeng Y, Vladar AE (2018) Metrology for the next generation of semiconductor devices. Nat Electron 1:532-547

47. Yacoot A, Koenders L (2011) Recent developments in dimensional nanometrology using AFMs. Meas Sci Technol 22:122001

48. Misumi I, Gonda S, Kurosawa T, Takamasu K (2003) Uncertainty in pitch measurements of one-dimensional grating standards using a nanometrological atomic force microscope. Meas Sci Technol 14:463-471

49. Misumi I, Gonda S, Huang Q, Keem T, Kurosawa T, Fujii A, Hisata N, Yamagishi T, Fujimoto H, Enjoji K, Aya S, Sumitani H (2005) Sub-hundred nanometre pitch measurements using an AFM with differential laser interferometers for designing usable lateral scales. Meas Sci Technol 16:2080-2090

50. Dixson R, Chernoff DA, Wang S, Vorburger TV, Tan SL, Orji NG (2010) Fu J (2010) Interlaboratory comparison of traceable atomic force microscope pitch measurements. Scanning Microsc 7729:77290M

51. Dai G, Koenders L, Pohlenz F, Dziomba T, Danzebrink HU (2005) Accurate and traceable calibration of one-dimensional gratings. Meas Sci Technol 16:1241-1249

52. Leach RK, Claverley J, Giusca C, Jones CW, Nimishakavi L, Sun W, Tedaldi M, Yacoot A (2012) Advances in engineering nanometrology at the National Physical Laboratory. Meas Sci Technol 23:074002

53. Misumi I, Gonda S, Sato O, Sugawara K, Yoshizaki K, Kurosawa T, Takatsuji T (2006) Nanometric lateral scale development using an atomic force microscope with directly traceable laser interferometers. Meas Sci Technol 17:2041-2047

54. Jäger G, Manske E, Hausotte T, Müller A, Balzer F (2016) Nanopositioning and nanomeasuring machine NPMM-200 - a new 
powerful tool for large-range micro- and nanotechnology. Surf Topogr Metrol Prop 4:034004

55. Misumi I, Sugawara K, Kizu R, Hirai A, Gonda S (2019) Extension of the range of profile surface roughness measurements using metrological atomic force microscope. Precis Eng 56:321-329

56. Gao W, Aoki J, Ju B-F, Kiyono S (2007) Surface profile measurement of a sinusoidal grid using an atomic force microscope on a diamond turning machine. Precis Eng 31:304-309

57. Dai G, Zhu F, Fluegge J (2015) High-speed metrological large range AFM. Meas Sci Technol 26:095402

58. Klapetek P, Picco L, Payton O, Yacoot A, Miles M (2013) Error mapping of high-speed AFM systems. Meas Sci Technol 24:025006

59. Quan L, Shimizu Y, Xin X, Matsukuma H, Gao W (2021) A new method for evaluation of the pitch deviation of a linear scale grating by an optical angle sensor. Precis Eng 67:1-13

60. Jaroszewicz Z (1986) Interferometric testing of the spacing error of a plane diffraction grating. Opt Commun 60:345-349

61. Gao W, Kimura A (2010) A fast evaluation method for pitch deviation and out-of-flatness of a planar scale grating. CIRP Ann Manuf Technol 59:505-508

62. Hecht E (2017) Optics, 5th edn. Pearson, London

63. Zhu X, Li X, Zhou Q, Wang X, Ni K (2016) A Blu-Ray laser diode based dual-beam interference lithography for fabrication of diffraction gratings for surface encoders. Adv Laser Process Manuf 10018:100180A

64. Li X, Shimizu Y, Ito SS, Gao W (2013) Fabrication of scale gratings for surface encoders by using laser interference lithography with $405 \mathrm{~nm}$ laser diodes. Int J Precis Eng Manuf 14:1979-1988

65. Neumann DB, Rose HW (1967) Improvement of recorded holographic fringes by feedback control. Appl Opt 6:1097

66. Bartolini RA (1974) Characteristics of relief phase holograms recorded in photoresists. Appl Opt 13:129

67. Kodate K, Kamiya T, Takenaka H, Yanai H (1978) Analysis of two-dimensional etching effect on the profiles of fine holographic grating made of positive photoresist AZ2400. Jpn J Appl Phys 17:121-126

68. Britten JA (1995) In situ end-point detection during development of submicrometer grating structures in photoresist. Opt Eng 34:474

69. Leclere P, Renotte Y, Lion Y (1992) Measure of the diffraction efficiency of a holographic grating created by two Gaussian beams. Appl Opt 31:4725

70. Shore BW, Perry MD, Britten JA, Boyd RD, Feit MD, Nguyen HT, Chow R, Loomis GE, Li L (1997) Design of high-efficiency dielectric reflection gratings. J Opt Soc Am A 14:1124

71. Ma D, Zhao Y, Zeng L (2017) Achieving unlimited recording length in interference lithography via broad-beam scanning exposure with self-referencing alignment. Sci Rep 7:1-10

72. Lloyd H (1834) On a new case of interference of the rays of light. Trans R Irish Acad 17:171

73. Titchmarsh PF (1941) Lloyd's single-mirror interference fringes. Proc Phys Soc 53:391-402

74. Xie Q, Hong MH, Tan HL, Chen GX, Shi LP, Chong TC (2008) Fabrication of nanostructures with laser interference lithography. J Alloys Compd 449:261-264

75. Huang CY, Ku HM, Chao S (2010) Fabrication of three-dimensional auto-cloned photonics crystal on sapphire substrate. Opt InfoBase Conf Pap 50:1-4

76. Li X, Shimizu Y, Ito S, Gao W, Zeng L (2013) Fabrication of diffraction gratings for surface encoders by using a Lloyd's mirror interferometer with a $405 \mathrm{~nm}$ laser diode. Proc SPIE Int Soc Opt Eng 8759:87594Q

77. Buitrago E, Fallica R, Fan D, Kulmala TS, Vockenhuber M, Ekinci Y (2016) SnOx high-efficiency EUV interference lithography gratings towards the ultimate resolution in photolithography. Microelectron Eng 155:44-49

78. Chen CG, Konkola PT, Heilmann RK, Joo C, Schattenburg ML (2002) Nanometer-accurate grating fabrication with scanning beam interference lithography. Nano Microtechnol Mater Process Packag Syst 4936:126

79. He J, Lin Y, Zhang X (2014) Fiber-based flexible interference lithography for photonic nanopatterning. Opt Express 22:26386

80. Shi L, Zeng L, Li L (2009) Fabrication of optical mosaic gratings with phase and attitude adjustments employing latent fringes and a red-wavelength dual-beam interferometer. Opt Express 17:21530

81. Shimizu Y, Aihara R, Ren Z, Chen Y-L, Ito S, Gao W (2016a) Influences of misalignment errors of optical components in an orthogonal two-axis Lloyd's mirror interferometer. Opt Express 24:18778-18789

82. Stay JL, Gaylord TK (2008) Three-beam-interference lithography: contrast and crystallography. Appl Opt 47:3221-3230

83. Kondo T, Matsuo S, Juodkazis S, Mizeikis V, Misawa H (2003) Multiphoton fabrication of periodic structures by multibeam interference of femtosecond pulses. Appl Phys Lett $82: 2758-2760$

84. Chua JK, Murukeshan VM (2009) Patterning of two-dimensional nanoscale features using grating-based multiple beams interference lithography. Phys Scr 80:015401

85. Stankevičius E, Daugnoraitė E, Račiukaitis G (2019) Mechanism of pillars formation using four-beam interference lithography. Opt Lasers Eng 116:41-46

86. Solak HH, David C, Gobrecht J, Wang L, Cerrina F (2002) Four-wave EUV interference lithography. Microelectron Eng 61-62:77-82

87. Berger V, Gauthier-Lafaye O, Costard E (1997) Photonic band gaps and holography. J Appl Phys 82:60-64

88. Li X, Gao W, Shimizu Y, Ito S (2014) A two-axis Lloyd's mirror interferometer for fabrication of two-dimensional diffraction gratings. CIRP Ann Manuf Technol 63:461-464

89. He J, Fang X, Lin Y, Zhang X (2015) Polarization control in flexible interference lithography for nano-patterning of different photonic structures with optimized contrast. Opt Express 23:11518

90. Vala M, Homola J (2014) Flexible method based on four-beam interference lithography for fabrication of large areas of perfectly periodic plasmonic arrays. Opt Express 22:18778

91. de Boor J, Geyer N, Gösele U, Schmidt V (2009) Three-beam interference lithography: upgrading a Lloyd's interferometer for single-exposure hexagonal patterning. Opt Lett 34:1783

92. Zhou H, Zeng L (2016) Method to fabricate orthogonal crossed gratings based on a dual Lloyd's mirror interferometer. Opt Commun 360:68-72

93. Solak HH, David C, Gobrecht J, Golovkina V, Cerrina F, Kim SO, Nealey PF (2003) Sub-50 nm period patterns with EUV interference lithography. Microelectron Eng 67-68:56-62

94. Li X, Lu H, Zhou Q, Wu G, Ni K, Wang X (2018) An orthogonal type two-axis Lloyd's mirror for holographic fabrication of two-dimensional planar scale gratings with large area. Appl Sci $8: 2283$

95. Shimizu Y, Aihara R, Ren Z, Chen Y-LY-L, Ito SS, Gao W (2016b) Influences of misalignment errors of optical components in an orthogonal two-axis Lloyd's mirror interferometer. Opt Express 24:27521

96. Chen X, Ren Z, Shimizu Y, Chen Y, Gao W (2017) Optimal polarization modulation for orthogonal two-axis Lloyd's mirror interference lithography. Opt Express 25:22237 
97. Chen X, Shimizu Y, Chen C, Chen Y-L, Gao W (2018) Generalized method for probing ideal initial polarization states in multibeam Lloyd's mirror interference lithography of 2D scale gratings. J Vac Sci Technol B Nanotechnol Microelectron Mater Process Meas Phenom 36:021601

98. Shimizu Y, Aihara R, Mano K, Chen C, Chen Y-L, Chen X, Gao W (2018) Design and testing of a compact non-orthogonal twoaxis Lloyd's mirror interferometer for fabrication of large-area two-dimensional scale gratings. Precis Eng 52:138-151

99. Cai Y, Li X, Aihara R, Zongwei R, Shimizu Y, Ito S, Gao W (2016) Investigation on the three-dimensional light intensity distribution of the fringe patterns generated by a modified twoaxis Lloyd's mirror interferometer. J Adv Mech Des Syst Manuf 10:JAMDSM0080

100. Shimizu Y, Mano K, Zhang K, Matsukuma H, Gao W (2019) Accurate polarization control in nonorthogonal two-axis Lloyd's mirror interferometer for fabrication of two-dimensional scale gratings. Opt Eng 58:1

101. Shimizu Y, Mano K, Murakami H, Hirota S, Matsukuma H, Gao W (2019) Design optimization of a non-orthogonal twoaxis Lloyd's mirror interferometer for fabrication of large-area two-dimensional scale gratings. Precis Eng 60:280-290

102. Hung Y-J, Chang H-J, Chang P-C, Lin J-J, Kao T-C (2017) Employing refractive beam shaping in a Lloyd's interference lithography system for uniform periodic nanostructure formation. J Vac Sci Technol B Nanotechnol Microelectron Mater Process Meas Phenom 35:030601

103. Shimizu Y, Kudo Y, Chen Y-LL, Ito S, Gao W (2017) An optical lever by using a mode-locked laser for angle measurement. Precis Eng 47:72-80

104. Shimizu Y, Matsukuma H, Gao W (2020) Optical angle sensor technology based on the optical frequency comb laser. Appl Sci 10:4047

105. Chen Y-L, Shimizu Y, Tamada J, Kudo Y, Madokoro S, Nakamura K, Gao W (2017) Optical frequency domain angle measurement in a femtosecond laser autocollimator. Opt Express 25:16725-16738

106. Chen Y-L, Shimizu Y, Kudo Y, Ito S, Gao W (2016) Modelocked laser autocollimator with an expanded measurement range. Opt Express 24:15554

107. Shimizu Y, Ishizuka R, Mano K, Kanda Y, Matsukuma H, Gao W (2021) An absolute surface encoder with a planar scale grating of variable periods. Precis Eng 67:36-47

108. Miyake M, Chen YC, Braun PV, Wiltzius P (2009) Fabrication of three-dimensional photonic crystals using muitibeam interference lithography and electrodeposition. Adv Mater 21:3012-3015

109. Divliansky I, Mayer TS, Holliday KS, Crespi VH (2003) Fabrication of three-dimensional polymer photonic crystal structures using single diffraction element interference lithography. Appl Phys Lett 82:1667-1669

110. Campbell M, Sharp DN, Harrison MT, Denning RG, Turberfield AJ (2000) Fabrication of photonic crystals for the visible spectrum by holographic lithography. Nature 404:53-56

111. Yang S, Chen G, Megens M, Ullal CK, Han Y-J, Rapaport R, Thomas EL, Aizenberg J (2005) Functional biomimetic microlens arrays with integrated pores. Adv Mater 17:435-438

112. Jeon S, Park JU, Cirelli R, Yang S, Heitzman CE, Braun PV, Kenis PJA, Rogers JA (2004) Fabricating complex three-dimensional nanostructures with high-resolution conformable phase masks. Proc Natl Acad Sci USA 101:12428-12433

113. Li W, Marconi MC (2015) Extreme ultraviolet Talbot interference lithography. Opt Express 23:25532

114. Kim M-S, Scharf T, Menzel C, Rockstuhl C, Herzig HP (2012) Talbot images of wavelength-scale amplitude gratings. Opt Express 20:4903
115. Chang CH, Tian L, Hesse WR, Gao H, Choi HJ, Kim JG, Siddiqui M, Barbastathis G (2011) From two-dimensional colloidal self-assembly to three-dimensional nanolithography. Nano Lett 11:2533-2537

116. Nakanishi H, Shinozaki M, Mizutani Y, Takaya Y (2019) Fabrication of 3D nano-periodic structure using multiple exposure lithography by Talbot effect. J Jpn Soc Precis Eng 85:710-716

117. Ito T, Okazaki S (2000) Pushing the limits of lithography. Nature 406:1027-1031

118. Martinez-Anton JC (2006) Surface relief subwavelength gratings by means of total internal reflection evanescent wave interference lithography. J Opt A Pure Appl Opt 8:S213-S218

119. Bezus EA, Doskolovich LL, Kazanskiy NL (2011) Evanescentwave interferometric nanoscale photolithography using guidedmode resonant gratings. Microelectron Eng 88:170-174

120. Masui S, Torii Y, Michihata M, Takamasu K, Takahashi S (2019) Fabrication of nano/micro dual-periodic structures by multibeam evanescent wave interference lithography using spatial beats. Opt Express 27:31522

121. Takahashi S, Masui S, Michihata M, Takamasu K (2020) Advanced generation of functional dual-periodic microstructured surface based on optical in-process measurement. CIRP Ann 69:477-480

122. Kong D, Michihata M, Takamasu K, Takahashi S (2018) Inprocess measurement of Resin's curing degree in micro-stereolithography using internal reflection at critical angle. J Phys Conf Ser 1065:142024

123. Huerta-Murillo D, Aguilar-Morales AI, Alamri S, Cardoso JT, Jagdheesh R, Lasagni AF, Ocaña JL (2017) Fabrication of multiscale periodic surface structures on Ti-6Al-4V by direct laser writing and direct laser interference patterning for modified wettability applications. Opt Lasers Eng 98:134-142

124. Weichert C, Bosse H, Flügge J, Köning R, Köchert P, Wiegmann A, Kunzmann H (2016) Implementation of straightness measurements at the nanometer comparator. CIRP Ann Manuf Technol 65:507-510

125. Sawabe M, Maeda F, Yamaryo Y, Simomura T, Saruki Y, Kubo T, Sakai H, Aoyagi S (2004) A new vacuum interferometric comparator for calibrating the fine linear encoders and scales. Precis Eng 28:320-328

126. Köning R, Weichert C, Przebierala B, Flügge J, Haessler-Grohne $\mathrm{W}$, Bosse H (2012) Implementing registration measurements on photomasks at the nanometer comparator. Meas Sci Technol 23:094010

127. Weichert C, Bütefisch S, Köning R, Flügge J (2017) Integration of a step gauge measurement capability at the PTB nanometer comparator-concept and preliminary tests. MacroScale website papers. https://doi.org/10.7795/810.20180323G

128. Krüger J, Köning R, Bodermann B (2020) Characterization progress of a UV-microscope recently implemented at the PTB Nanometer comparator for uni- and bidirectional measurements. EPJ Web Conf 238:06010

129. Heidenhain (2016) Exposed_linear_encoders. Heidenhain. https ://www.heidenhain.com/

130. Bosse H, Wilkening G (2005) Developments at PTB in nanometrology for support of the semiconductor industry. Meas Sci Technol 16:2155-2166

131. Shimizu Y, Uehara K, Matsukuma H, Gao W (2018) Evaluation of the grating period based on laser diffraction by using a modelocked femtosecond laser beam. J Adv Mech Des Syst Manuf 12:JAMDSM0097

132. Buhr E, Michaelis W, Diener A, Mirandé W (2007) Multi-wavelength VIS/UV optical diffractometer for high-accuracy calibration of nano-scale pitch standards. Meas Sci Technol 18:667-674 
133. Korpelainen V, Iho A, Seppä J, Lassila A (2009) High accuracy laser diffractometer: angle-scale traceability by the error separation method with a grating. Meas Sci Technol 20:084020

134. Decker JE, Eves BJ, Pekelsky JR, Douglas RJ (2011) Evaluation of uncertainty in grating pitch measurement by optical diffraction using Monte Carlo methods. Meas Sci Technol 22:027001

135. Misumi I, Gonda S, Kurosawa T, Tanimura Y, Ochiai N, Kitta J, Kubota F, Yamada M, Fujiwara Y, Nakayama Y, Takamasu K (2003) Submicrometre-pitch intercomparison between optical diffraction, scanning electron microscope and atomic force microscope. Meas Sci Technol 14:2065-2074

136. Cocco D, Sostero G, Zangrando M (2003) Technique for measuring the groove density of diffraction gratings using the long trace profiler. Rev Sci Instrum 74:3544-3548

137. Lim J, Rah S (2004) Technique for measuring the groove density of a diffraction grating with elimination of the eccentricity effect. Rev Sci Instrum 75:780-782

138. Liu B, Lou J, Fu SJ, Xu XD, Wang QP (2006) Two-dimensional measurement of groove spacing for plane VLS gratings using the long trace profiler. In: 2006 1st IEEE international conference on nano/micro engineered and molecular systems. IEEE, pp $556-558$

139. Ennos AE, Virdee MS (1982) High accuracy profile measurement of quasi-conical mirror surfaces by laser autocollimation. Precis Eng 4:5-8

140. Takacs PZ (2003) Equal optical path beamsplitter for a pencil beam interferometer and shearing interferometer. Opt Eng 42:929

141. Gao W (2010) Precision nanometrology. Springer, London

142. Shimizu Y, Tan SL, Murata D, Maruyama T, Ito S, Chen Y-L, Gao W (2016) Ultra-sensitive angle sensor based on laser autocollimation for measurement of stage tilt motions. Opt Express 24:2788

143. Saito Y, Gao W, Kiyono S (2007) A single lens micro-angle sensor. Int J Precis Eng Manuf 8:14-18

144. Gao W, Ohnuma T, Satoh H, Shimizu H, Kiyono S, Makino H (2004) A precision angle sensor using a multi-cell photodiode array. CIRP Ann Manuf Technol 53:425-428

145. Gao W, Saito Y, Muto H, Arai Y, Shimizu Y (2011) A three-axis autocollimator for detection of angular error motions of a precision stage. CIRP Ann Manuf Technol 60:515-518

146. Saito Y, Arai Y, Gao W (2009) Detection of three-axis angles by an optical sensor. Sens Actuat A Phys 150:175-183

147. Voronov DL, Diez S, Lum P, Hidalgo SA, Warwick T, Artemiev N, Padmore HA (2013) Fabrication of X-ray gratings by direct write maskless lithography. In: Khounsary A, Goto S, Morawe $\mathrm{C}$ (eds) Advances in X-ray/EUV optics and components, vol 8. SPIE, Washington, $\mathrm{p} 88480 \mathrm{Q}$

148. Xiong X, Matsukuma H, Shimizu Y, Gao W (2020) Evaluation of the pitch deviation of a linear scale based on a self-calibration method with a Fizeau interferometer. Meas Sci Technol 31:094002 (in press)

149. Chen X, Shimizu Y, Xiong X, Chen Y-LY, Gao W (2017) Selfcalibration of Fizeau interferometer and planar scale gratings in Littrow setup. Opt Express 25:21567

150. Xiong X, Shimizu Y, Chen X, Matsukuma H, Gao W (2018) Uncertainty evaluation for measurements of pitch deviation and out-of-flatness of planar scale gratings by a Fizeau interferometer in Littrow configuration. Appl Sci 8:2539 